\title{
Forging of Aluminium Components under a Superimposed Hydrostatic Pressure to Induce Local Strain Hardening
}

\author{
Bernd-Arno Behrens ${ }^{1}$, Thomas Hagen ${ }^{1, a}$, Andreas Klassen ${ }^{1, b}$, \\ Julian Knigge ${ }^{1, \mathrm{c}}$, Jens Mielke ${ }^{1, \mathrm{~d}}$ and Insa Pfeiffer ${ }^{1, \mathrm{e}}$ \\ ${ }^{1}$ Institute of Metal Forming and Metal-Forming Machines (IFUM), \\ Leibniz Universität Hannover (LUH), An der Universität 2, \\ 30823 Garbsen, Germany \\ ahagen@ifum.uni-hannover.de, ${ }^{b}$ klassen@ifum.uni-hannover.de, ${ }^{c}$ knigge@ifum.uni-hannover.de, \\ dmielke@ifum.uni-hannover.de, ${ }^{\mathrm{e}}$ pfeiffer@ifum.uni-hannover.de
}

Keywords: strain hardening, cold forging, active means, superimposed hydrostatic pressure, simulation, damage prediction

\begin{abstract}
A promising approach to handling low ductile aluminium alloys in a forming process is forming under superimposed hydrostatic pressure. The influence of superimposed hydrostatic pressure on the flow stress as well as on the formability for various hydrostatic pressures and temperatures was analysed [15, 3, and 7]. By increasing the formability of the workpiece, larger local plastic strains could be achieved. The results reveal highly increased formability at superimposed pressure of $85 \mathrm{MPa}$ for workpieces from thermosetting alloy AlSi1MgMn (EN AW-6082) in comparison to those from self-hardening alloy AlMg4.5Mn0.7 (EN AW-5083). As a general tendency, the self-hardening alloys show a lower increase in formability when forged under superimposed pressure. But additionally, a charge-dependent influence of macro- and micro defects on the crack resistance was detected for alloy AlMg4.5Mn0.7. By evaluating damage models in finite element models the damage occurring in cold forming processes under superimposed hydrostatic pressure was predicted.
\end{abstract}

\section{Introduction}

Conventional forging processes often offer only low performances for modern high-strength materials or PM aluminium alloys. Low material formability results in cracking during the forming process. The material forming limit in a forming process depends on the stress state as Pawelski noted in [17]. As a rule, regarding the forming capability forming processes under compressive stress states are superior to those under tensile stress conditions. Therefore, a forging tool was designed to superimpose a hydrostatic pressure during the forming operation. An essential research topic was to analyse the influence of a superimposed hydrostatic pressure on the formability as well as on the flow stress for different procedures at different hydrostatic pressures and temperature levels [15, 3, and 7]. It was observed that flow stress is independent of superimposed hydrostatic pressure. Neither does the superimposed pressure have an influence on the plastic deformation, nor does a pressure-dependent material hardening due to increasing hydrostatic pressure take place. Nevertheless, the formability increased with rising hydrostatic pressure. The lower the forming temperatures, the higher the value of superimposed pressure has to be to gain a significant increase in formability. Therefore, in this paper a tool concept is described, which offers a superimposed hydrostatic pressure of maximum $200 \mathrm{MPa}$. The increase in formability is used to induce high local plastic strains. These lead to an increase in strain hardening and through this to higher component strength and hardness.

Consequently, different aluminium alloys (thermosetting as well as self-hardening alloys) were characterised and then forged under superimposed pressure to determine the increase in local strain hardening. 
Supporting the tool design process FEA was used to compute the forging process with various parameters such as different forming geometries, amounts of superimposed pressure and different aluminium alloys. Especially, the components of the tool system highly stressed due to internal pressure of $200 \mathrm{MPa}$ were verified. Furthermore, by implementing damage models into the FEA model reliable damage prediction for the forging process under superimposed pressure was achieved.

\section{Design of a tool concept for cold forging under superimposed pressure}

Based on preliminary research [15], an increase in superimposed pressure up to $200 \mathrm{MPa}$ is considered to be required for forming complex workpieces of low ductile materials at room temperature. Therefore, a new tool concept equipped with a parted die and dimensioned for high superimposed pressures was developed. During the design process two types of closing systems were elaborated, as displayed in Fig. 1 and Fig. 2.

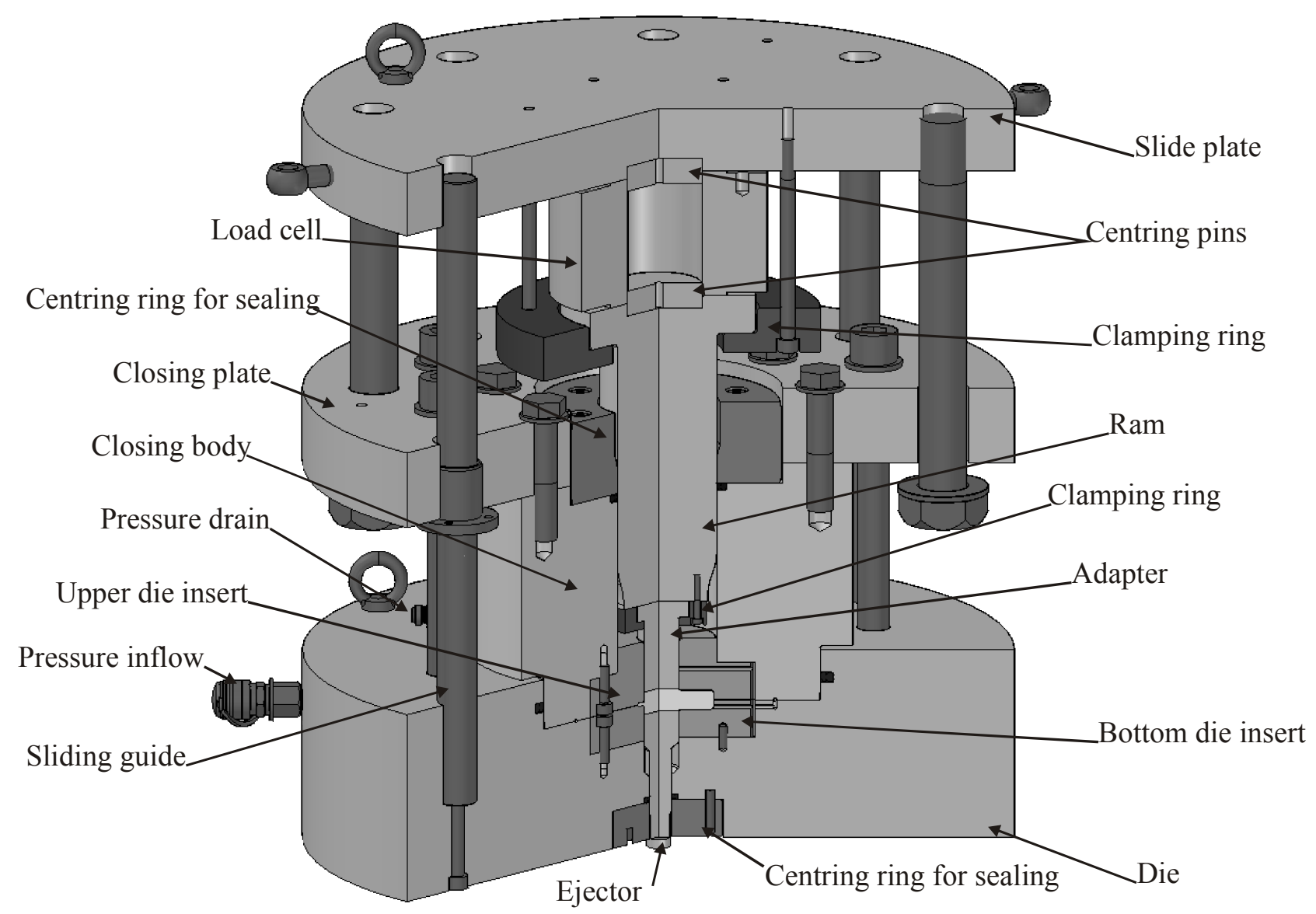

Fig. 1: Tool concept for superimposed pressures up to $200 \mathrm{MPa}$ with bolted fastening closing plate 


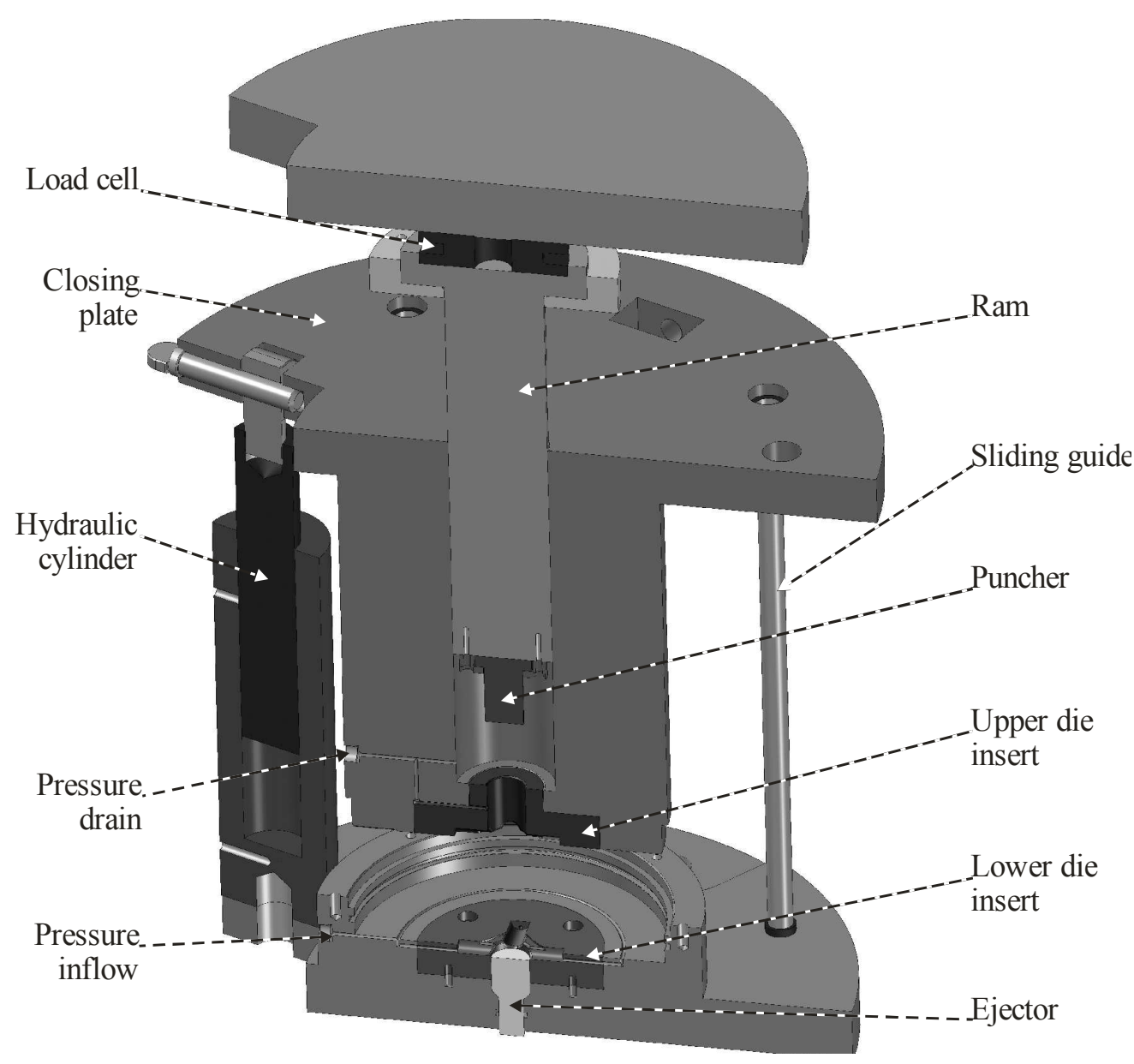

Fig. 2: Tool concept for superimposed pressures up to $200 \mathrm{MPa}$ with hydraulically fastened closing plate

Of crucial importance is the application of the required closing pressure for a safe sealing of the internal pressure. Within the tool system in Fig. 1 the closing plate is fastened onto the die by screws. This is an easy way to provide a high amount of closing pressure as demanded due to the internal pressure. But, for opening and closing the die system, the screws have to be dismantled for each test. Therefore, this concept lacks in usability considering serial production aspects, nevertheless it is useful for bench tests. Within the tool system in Fig. 2 the closing plate is fastened onto the die by 4 hydraulic cylinders, which allow easy opening/closing of the die system. The hydraulic closing system offers a convenient operability, but for acquiring a high safety level, the cylinders have to provide a mechanical clamp technique to utterly arrest in case of drive pressure loss to avoid leaking of the active media. Another way to arrest a parted die system is the use of compression springs. In this case for forming under superimposed pressure, using springs or spring discs is not useful, because the closing force is stroke-dependent and therefore a safe arresting of the closing plate while applying active pressure is impossible.

Because of its modular design the developed tool concept as shown in Fig. 3 offers a hydraulically driven as well as a screwed closing system. To switch the closing method only the closing plate has to be changed. The tool is dimensioned for maximum superimposed pressures of $200 \mathrm{MPa}$, die inserts with a maximum diameter of $85 \mathrm{~mm}$ (max. sealing diameter $115 \mathrm{~mm}$ ) resulting in a required closing force of $2100 \mathrm{kN}$. 


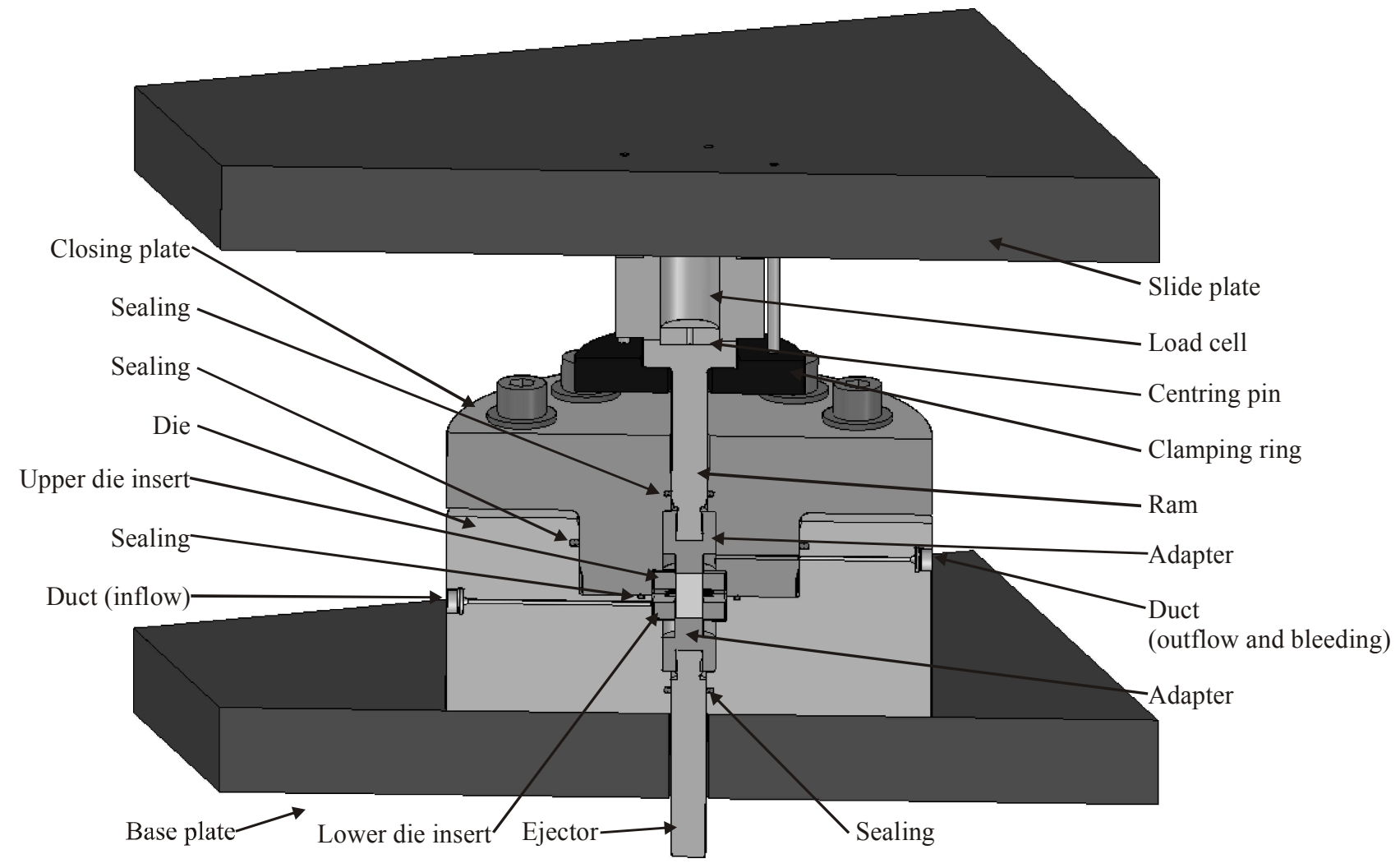

Fig. 3: Tool system for cold forging under a superimposed pressure of maximum $200 \mathrm{MPa}$

The modular design of this hydromechanical forming tool (Fig. 3) is prepared for small space requirements and enables easy-to-handle fitting into the forging press. The die system is mantled to the pillar guide frame to compensate slide tilting. The functionality of this tool system is equal to the tool concept displayed in Fig. 1. The cylindric sample is placed centrically onto the lower die insert when the closing plate is lifted and thus the tool system is open. The closing plate is moved down by the upper ram of the forging press and centred with the die and then screwed onto the die. Then the hydraulic pressure is provided by an active hydraulic power unit.

During the forming operation the medium drains back to the medium tank and the superimposed pressure is controlled by pressure valves. Upon reaching bottom dead centre the superimposed pressure is slowly reduced to zero pressure. Afterwards, the closing plate gets dismantled and subsequently the ram lifts the upper die system. The workpiece can be removed.

A wide range of workpiece geometries can be obtained by easy-to-handle die inserts. By supplying the media pressure through various sewers in $\mathrm{x}, \mathrm{y}, \mathrm{z}$-directions, the superimposed pressure can be obtained and maintained at the actual non-contacted surfaces during the forming operation. To determine the increase in strain hardening within this project various die inserts were designed, the most important being the die inserts for upsetting and lateral extrusion as shown in Fig. 4. The adapters of the inserts are prepared with a female thread and can be easily mounted either onto the ram or onto the ejector. The die inserts for lateral extrusion are clamped by a clearance fit and positioned by centring pins. On that account, no additional thread holes are required and the component profiles maintain their original strength. 


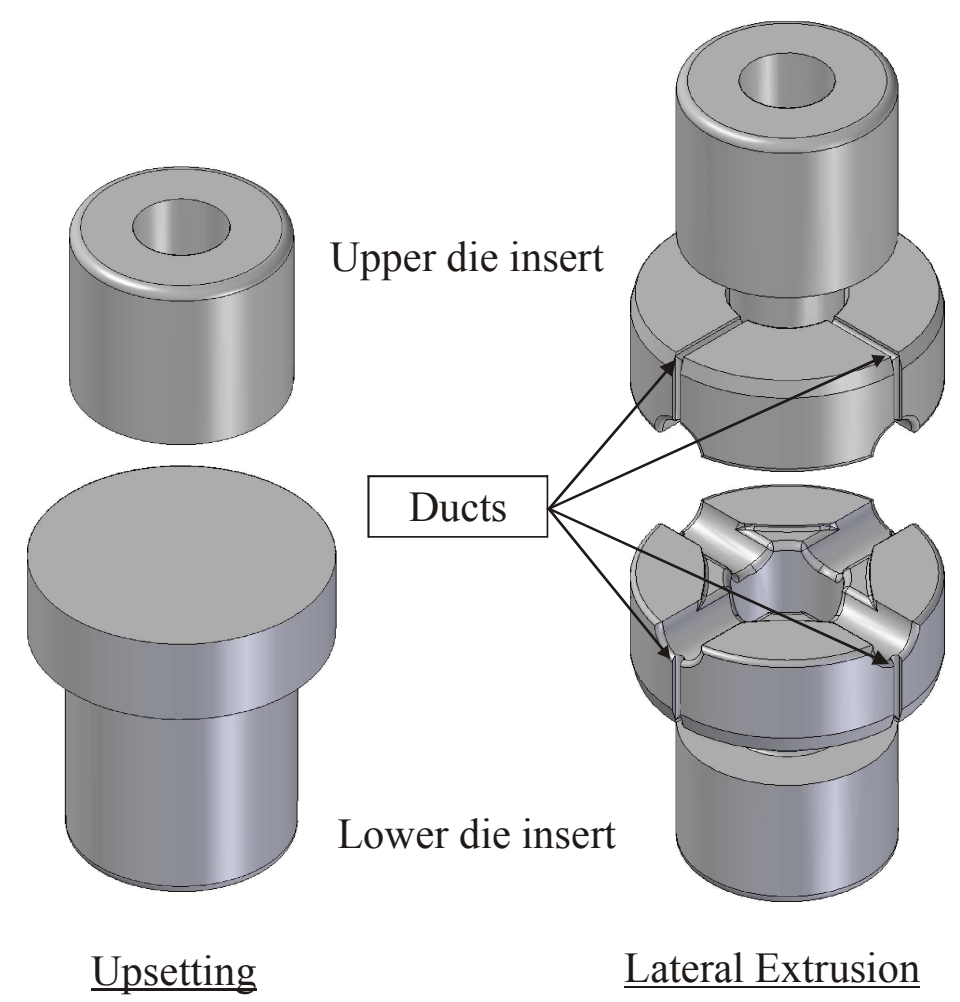

Fig. 4: Die inserts for upsetting (left) and lateral extrusion (right)

Forming processes with identical and faultless workpieces show the same force paths. Hence, occurring errors within the forming process can be detected by differing force paths. Therefore, a load cell is mounted beneath the slide plate to measure the axial force paths during forming operation. The stroke is measured by the transducer system integrated in the forging machine. The superimposed pressure is monitored by means of a pressure manometer with signal output mounted near the pressure drain of the die.

As sealings common Merkel Omegat OMS-MR sealings are used. They consist of a PTFE profile ring and an o-ring seal used for prestressing (see Fig. 5).

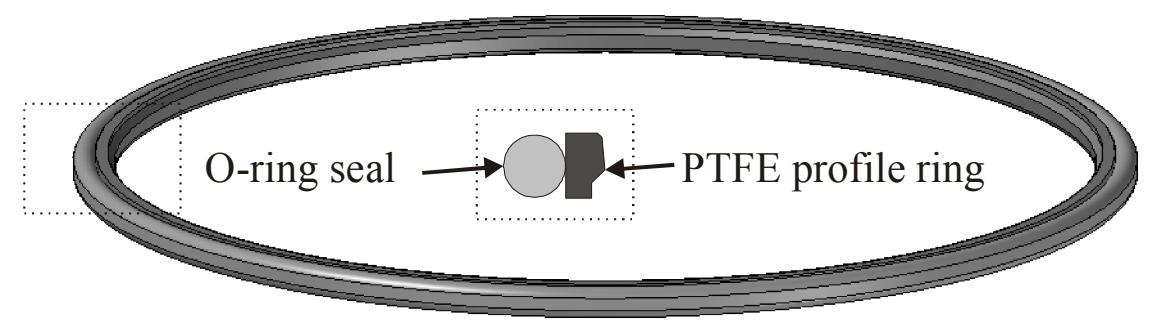

Fig. 5: Scheme of a typical Merkel Omegat OMS-MR sealing

The sealings are positioned in single rectangular slots. The durable PTFE profile ring provides dynamic sealing and the elastic o-ring implements static sealing and prestresses the profile ring. Seal effects are increased by the active system pressure. Commonly, standard hydraulic sealings are used for pressures of maximum $40 \mathrm{MPa}$. To increase this pressure limit, the clearance between the sealed components was set to maximum 50 microns and the surface durability of the components was increased by plasma nitriding.

To achieve good pressure controllability, the active medium must provide a constant pressure response during the test. Linear or volatile behaviour of the medium leads to limited adjustability of the pressure valves resulting in inadequate test repeatability. The medium has to resist pressures of 
maximum $250 \mathrm{MPa}$ and to solute little amounts of air without carbonising. In former tests with pressures up to $60 / 100 \mathrm{MPa}$ the following media were examined:

- castor oil

- Shell Tellus 46 HLP DIN 51524-2

- Hydrofluid HAT 42

- Renoform HFL 3135

- Zeller \& Gmelin MultiDraw HT400

As a result, all media supplied low compressibility and high viscosity. Besides, castor oil offered the best performance at low temperatures and max. $60 \mathrm{MPa}$ and is the most inexpensive medium. But, at higher temperatures and pressures above $50 \mathrm{MPa}$ MultiDraw HT400 showed the best viscosity and heat transfer. Considering the test setup with $200 \mathrm{MPa}$ superimposed pressure and room temperature, the hydraulic power unit used to pressurise the die system and the pressure controlling system as well require hydraulic oil with a viscosity of $46 \cdot 10^{-6} \mathrm{~mm}^{2} / \mathrm{s}$.

\section{Material characterisation}

By choosing various aluminium alloys with different attributes as listed in Table 1 a broad field of applications can be examined.

Table 1: Test materials for forging tests

\begin{tabular}{|c||c|c||c|}
\hline $\begin{array}{c}\text { Material name EN } \\
\text { AW / DIN }\end{array}$ & Alloy & Comment & Material condition: \\
\hline \hline $2017 \mathrm{~A} / 3.1325$ & $\begin{array}{c}\text { AlCu4MgSi (A) } \\
\text { (former AlCuMg1) }\end{array}$ & thermosetting & T4 EN 573-3 \\
\hline \hline $5083 / 3.3547$ & $\mathrm{AlMg} 4.5 \mathrm{Mn} 0.7$ & self-hardening & H112 EN 755-1,2,3 \\
\hline \hline $5754 / 3.3535$ & $\mathrm{AlMg}$ & self-hardening & H111 EN 573-1,2,3 \\
\hline $6082 / 3.2315$ & $\mathrm{AlSi1MgMn}$ & thermosetting & T6 EN 573-3 \\
\hline \hline $7075 / 3.4365$ & $\begin{array}{c}\text { AlZn5.5MgCu } \\
\text { (former AlZnMgCu1.5) }\end{array}$ & thermosetting & T6 EN 573-3 \\
\hline \hline \multicolumn{7}{|l|}{ T4-Solution heat treatment and natural ageing according to DIN EN 515/ DIN 29850 } \\
\hline T6-Solution heat treatment and artificial ageing according to DIN EN 515/ DIN 29850 \\
\hline
\end{tabular}

To characterise the mechanical properties of the test materials the following tests were carried out:

- Tensile tests

- Notched-bar impact test

- Cylinder compression tests for flow curve determination

- Ball indentation test (Hardness Brinell) test

The material parameters of the tensile tests as well as the flow curves were implemented into the used FEA-programs to acquire representable material data.

\section{Tensile Tests}

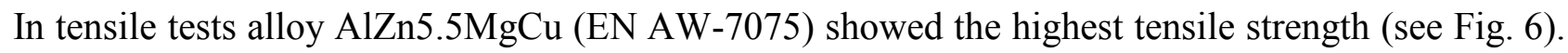
With an amount of $\sim 650 \mathrm{MPa}$ it is clearly on a major level compared to the other test alloys. But, the tensile test samples of $\mathrm{AlZn} 5.5 \mathrm{MgCu}$ showed nearly no reduction in area and therefore tend to extreme brittleness. The same behaviour was exhibited by alloy AlCu4MgSi (EN AW-2017A). On the contrary, the self-hardening alloys all showed good ductility. As expected $[10,5]$, the Portevin- 
Le Châtelier instability is observed for the Al-Mg alloys. It is persistent over nearly the entire plastic strain range until necking. This and the breaking surface appearance of the tensile test specimen indicate a coarse-grained structure for these two test materials. The characteristic values of the accomplished tensile tests are listed in Table 2.

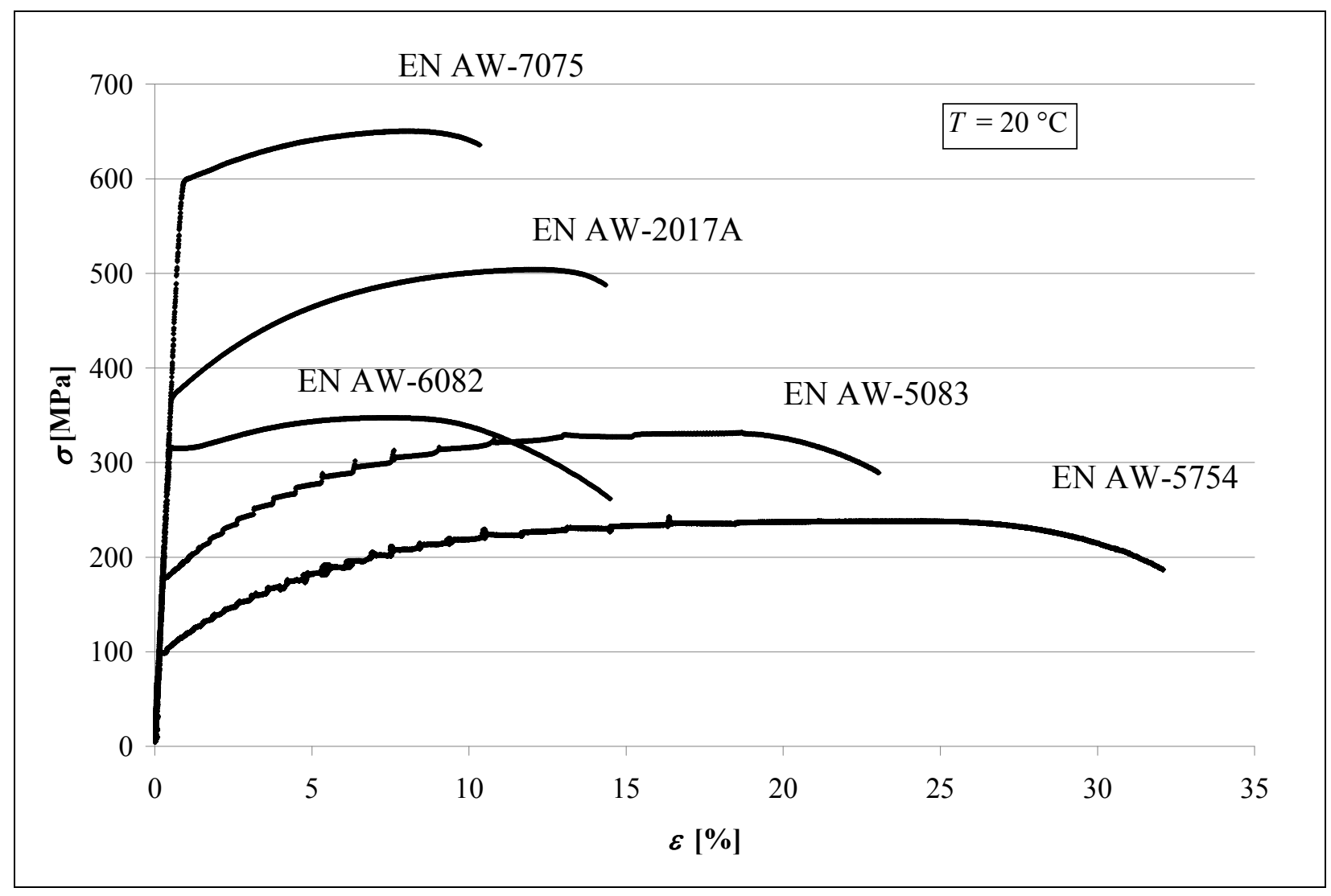

Fig. 6: Stress-strain curves of test materials obtained by tensile tests

Table 2: Characteristic test material parameters obtained by tensile tests

\begin{tabular}{|c|c|c|c|c|}
\hline $\begin{array}{c}\text { Material name } \\
\text { EN AW / DIN }\end{array}$ & $\begin{array}{c}\text { Yield stress } \\
R_{\mathrm{p} 0,2}[\mathrm{Mpa}]\end{array}$ & $\begin{array}{c}\text { Tensile strength } \\
R_{\mathrm{m}}[\mathrm{Mpa}]\end{array}$ & $\begin{array}{c}\text { Breaking elongation } \\
A[\%]\end{array}$ & $\begin{array}{c}\text { Reduction of } \\
\text { area } \\
Z[\%]\end{array}$ \\
\hline $7075 / 3.4365$ & 607.6 & 657 & 10.1 & - \\
\hline $2017 \mathrm{~A} / 3.1325$ & 375.2 & 504 & 14.7 & 44 \\
\hline $6082 / 3.2315$ & 315 & 348 & 15.3 & 44 \\
\hline $5083 / 3.3547$ & 182 & 333 & 24.8 & 58 \\
\hline $5754 / 3.3535$ & 99.2 & 237 & 31.1 & \\
\hline
\end{tabular}




\section{Determination of flow curves by means of a cylinder compression test}

Flow curves are essential to characterise the plastic material behaviour and give the coherence between flow stress $\mathrm{k}_{\mathrm{f}}$ and true strain $\varphi$. As a rule for forging processes the flow curve is obtained by cylinder compression tests. Here, a cylindric sample is upset by compression stresses on the cross sectional area. By applying sufficient lubrication to the end planes a uniaxial state of stress is assumed. For cold forging processes the influence of the strain rate on the flow stress is insignificant. But, regarding formability the faster the strain rate, the lower is the material forming limit. The flow curve is reliable as long as no cracking occurs. In Fig. 7 the results from the cylinder compression tests for the test alloys are shown.

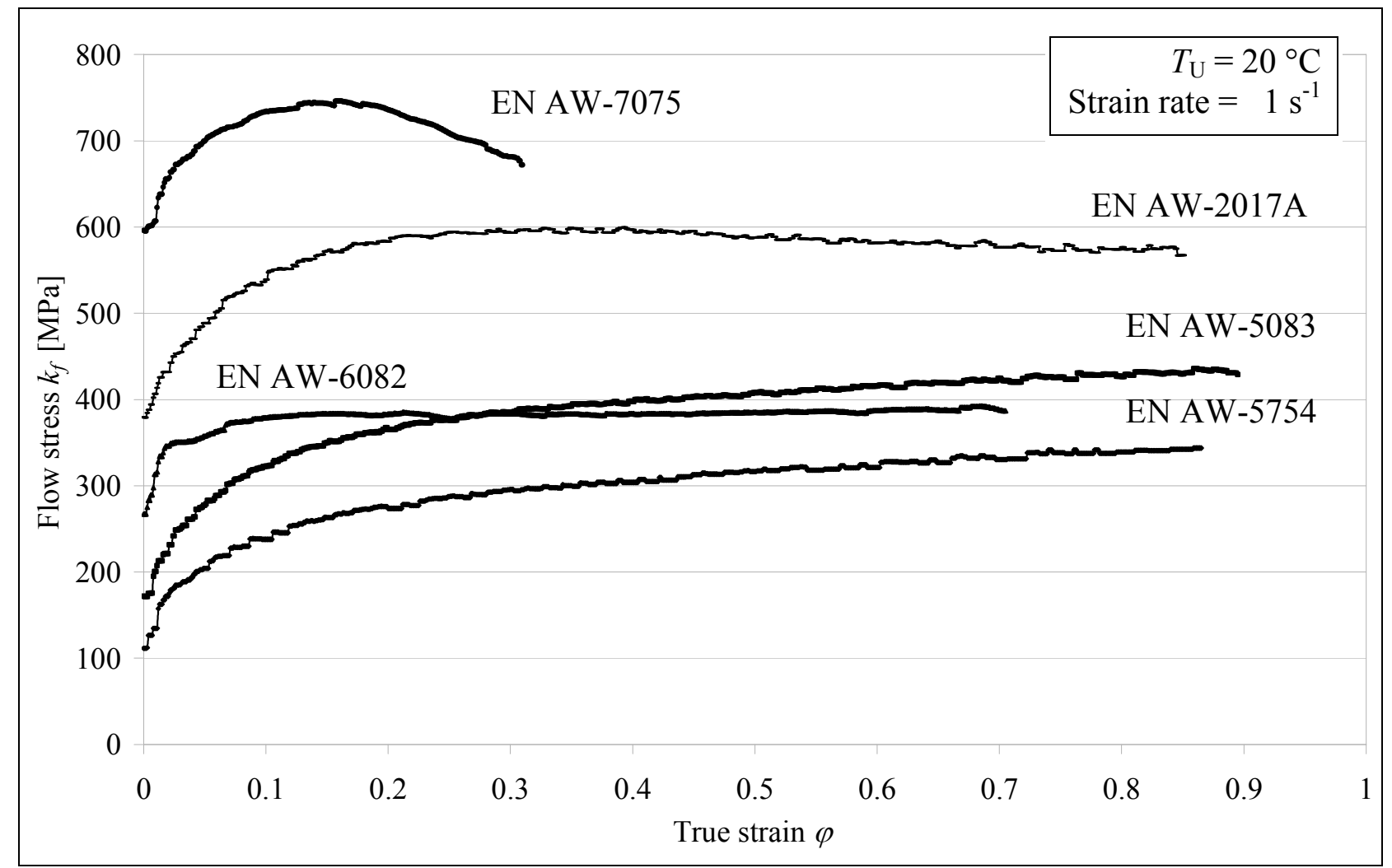

Fig. 7: Flow curves of the test materials obtained by cylinder compression tests

The flow stresses at yield strain correspond to those from the tensile tests mentioned in Table 2. For the alloys AlZn5.5MgCu (EN AW-7075) and AlSi1MgMn (EN AW-6082) cracks occurred before reaching the desired maximum true strain of 0.9 , so the flow curves were cropped to the crack ignition strain. Due to forming the self-hardening alloys AlMg4.5Mn0.7 (EN AW-5083) and AlMg3 (EN AW-5754) experienced continuous strain hardening. On the contrary, the flow curves of the thermosetting alloys show strain softening.

\section{Brinell Hardness tests}

Hardness is defined as the part's resistance against penetration of another part of higher hardness. According to the Brinell Hardness testing method (adequate to DIN 50351), the ball indentation diameter of a ball consisting of hard metal or hardened steel penetrating a test sample with a defined test load vertical to the test sample's surface is measured. As the ball indentation includes a relatively large area of the test surface, the ball indentation test is suitable for testing inhomogeneous materials. 
The hardness was measured at 2 points on each end plane of the raw pieces, near-edge and centre. The results are displayed in Fig. 8. The examined aluminium alloys feature a hardness range between $65 \mathrm{HB}$ and $196 \mathrm{HB}$. Alloy AlZn5.5MgCu (EN AW-7075) reveals the highest hardness values and AlMg3 (EN AW-5754) the lowest.

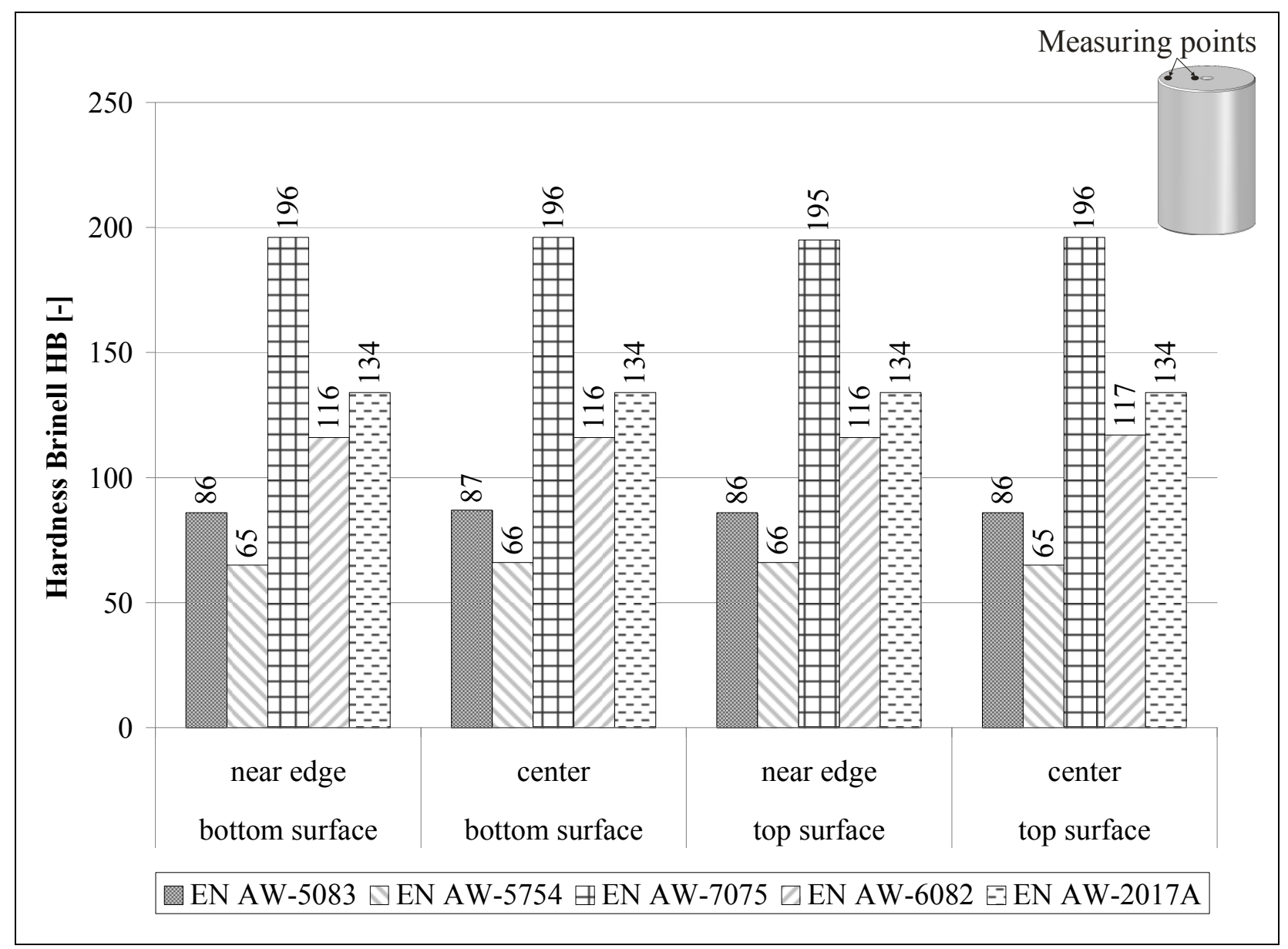

Fig. 8: Brinell Hardness of the raw piece

\section{Notched-bar impact bending test (according to method of Charpy)}

The notched-bar impact bending test is used to determine ductile values of the test materials and gives a hint of formability. According to Charpy's method, a sample beam, notched on one side and clamped between two bearings, is penetrated by a swing hammer with one single hit. The loss in potential energy is measured and the notch impact resistance is derived from the absorbed impact energy. The higher the notch impact resistance $A_{V}$, the higher the ductility.

The tests were carried out at the Institute of Materials Science of the Leibniz Universität Hannover at $20^{\circ} \mathrm{C}$ ambient test temperature. Test samples were consistent with ISO V-notch specimen adequate to DIN 50115. The test results are displayed in Fig. 9. The alloy AlZn5.5MgCu (EN AW-7075) shows very little notch resistance leading to a very brittle forming behaviour as already mentioned within the discussion of the tensile tests. The self-hardening alloys have the highest notch resistance values and showed ductile cracking. 


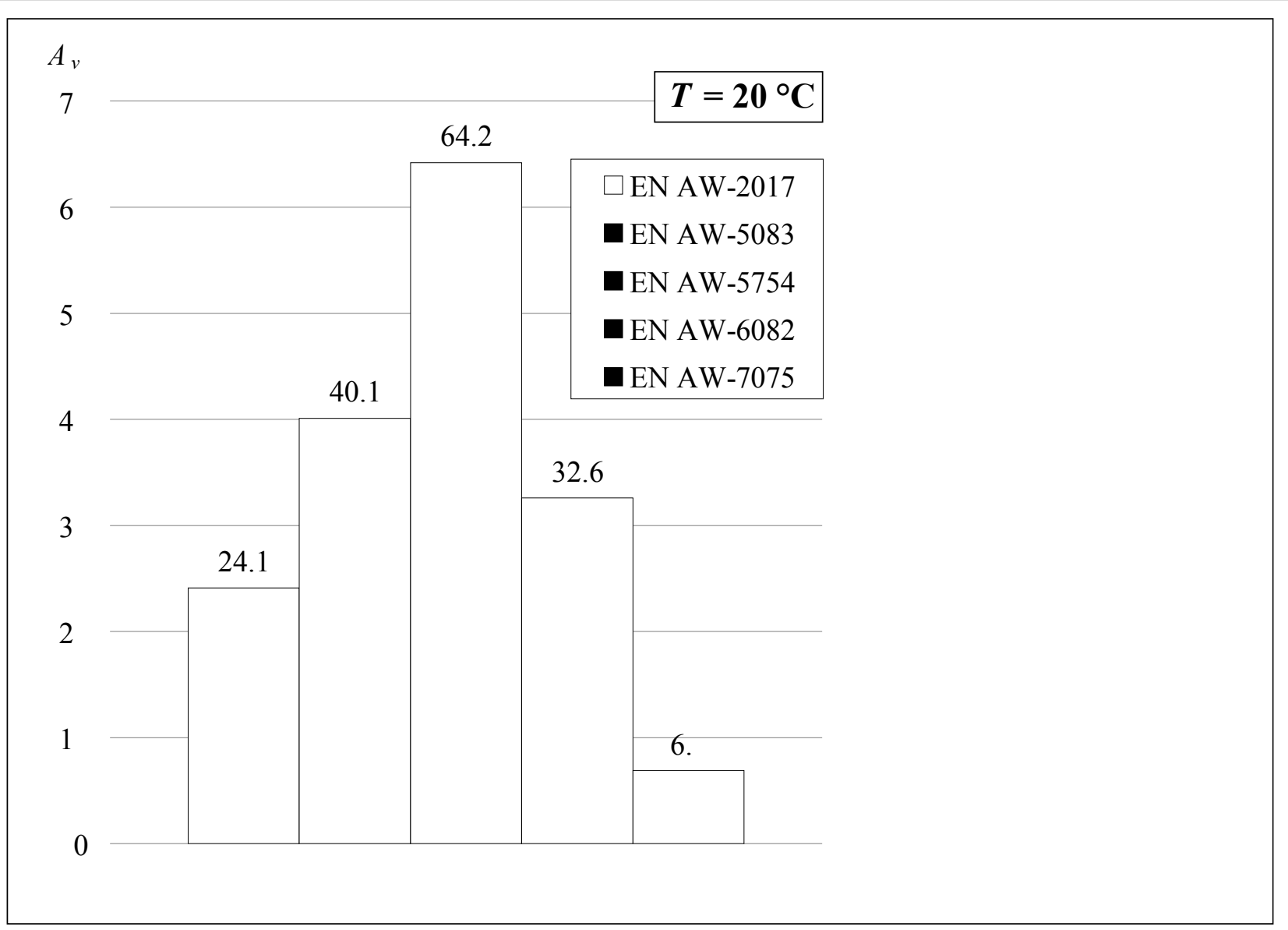

Fig. 9: Notch impact resistance of the test materials from notched-bar impact bending tests

\section{Upsetting under superimposed pressure}

To determine the influence of the superimposed pressure on formability and strain hardening various upsetting tests were carried out. Objectives were those process parameters ensuring a free of cracks forming with highest increase in local formability and least superimposed pressure depending on the considered alloy. For the upsetting tests the tool system illustrated in Fig. 1 was used. To reduce the amount of tests and to avoid equidistant stepping tests, the crack initiation as limit criterion of formability was detected by acoustic emission sensors (AE). The influence of the superimposed pressure within the upsetting process on the formability of the test materials was examined, as displayed in Fig. 10 and Fig. 11. 


\begin{tabular}{|r|c|c|}
\hline $\begin{array}{r}\text { AlSi1MgMn } \\
\text { Material condition: } \\
\begin{array}{r}\text { Superimposed } \\
\text { pressure: }\end{array}\end{array}$ & \multicolumn{2}{|c|}{ T6 } \\
\hline \\
\hline
\end{tabular}

Fig. 10: Influence of superimposed pressure on the formability of AlSi1MgMn (EN AW-6082) for

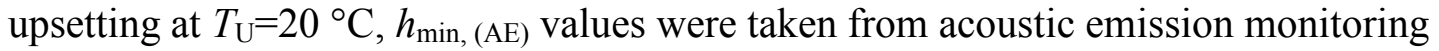

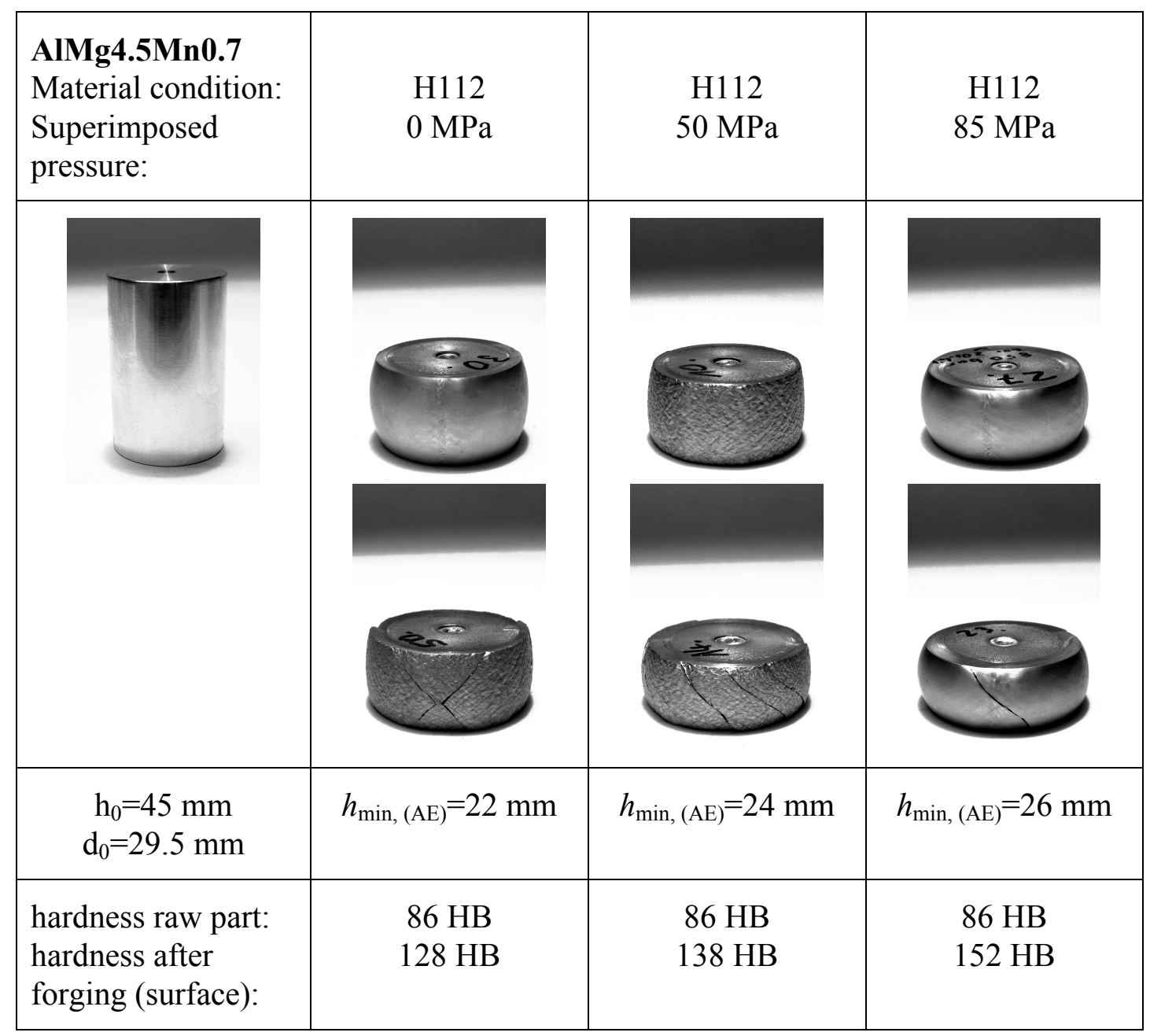

Fig. 11: Influence of superimposed pressure on the formability of AlMg4.5Mn0.7 (EN AW-5083)

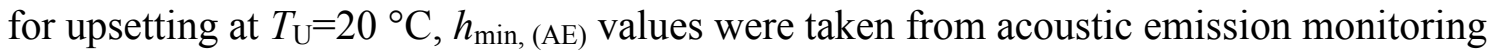


By increasing the formability of the workpiece, higher local plastic strains could be achieved. The results reveal highly increased formability at a superimposed pressure of $85 \mathrm{MPa}$ for workpieces from thermosetting alloy AlSilMgMn (EN AW-6082) in comparison to those from self-hardening alloy AlMg4.5Mn0.7 (EN AW-5083). As a general tendency, the self-hardening alloys show a lower increase in formability when forged under superimposed pressure. But additionally, a chargedependent influence of macro- and micro defects on the crack resistance was detected for alloy AlMg4.5Mn0.7, as shown in Fig. 12.

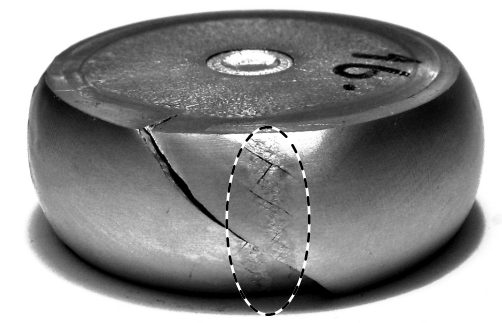

Fig. 12: Charge-dependent macro defects on the specimens of AlMg4.5Mn0.7 (EN AW-5083)

\section{FEA simulation of the forging process under superimposed pressure}

Using FEA for the design of forming processes allows minimising cost and time intensive empirical testing to verify and validate tool concepts and forming processes. Forming behaviour like material flow, form filling, forming laps and forming force can be predicted as well as thermal and mechanical loads of the tools. Moreover, the finite element analysis (FEA) is widely used within the optimisation and design of forging processes. Especially in the described forming process under superimposed hydrostatic pressure, the simulation-based process design is of high importance.

Here, the accurate description of the superimposed pressure and the exact knowledge about the process-related boundary conditions, the friction and the heat transfer are necessary in order to ensure high simulation accuracy. For the forming processes investigated in this paper the occurring friction conditions and the amount of the superimposed pressure are of special interest because these parameters strongly influence the stress-strain state in the surface of the billets. Moreover, the material behaviour of the workpiece is required in the form of flowcurves in order to obtain a realistic material flow simulation.

Preliminary research at the Institute of Metal Forming and Metal-Forming Machines revealed that by means of FEA a massive forming process with active media-based superimposed pressure can be simulated [2]. The superimposed pressure is applied through normal pressure on each surface element of the workpiece.

A subject-matter of this research project is the consideration and modelling of the strain hardening effect of the aluminium alloys under study. Hereby, the achievable amount of strain hardening depending on the local forming ratio, raw part geometry and microstructure state, superimposed pressure value and forming velocity can be estimated.

Experiments have already validated that the formability can be increased by means of active media and thus ductile damage in forging of light metals can be avoided. Therefore, it is also essential to develop a numerical model in order to simulate the forging process with superimposed hydrostatic pressure. In this simulation model parameters like pressure, flowcurve and geometry were varied. With the help of this simulation model it is possible to predict the ductile damage in the workpiece during the process and the local strain hardening effects can be shown.

Supporting the tool design process FEA was used to compute the forming process with various parameters such as different forming geometries, amounts of superimposed pressure and different aluminium alloys. 
Especially the effect of hydrostatic pressure on the ductile damage is one of the focal points in this study.The simulation results of the process simulation were validated by means of numerous experiments as shown before.

First the maximum loads of the tools were computed in order to optimise the tool geometry and the process parameters. Therefore, the cold forging process of a cross geometry was analysed using FEA to show a complete form filling and the stresses of the tool within acceptable range.

\section{Tool Design by FE-Analysis}

For the analysis of the lateral extrusion process, a round workpiece with a height of $53 \mathrm{~mm}$ and a diameter of $30 \mathrm{~mm}$ is formed by means of a toolset consisting of two oppositely arranged punches that move towards each other and a forming die. The material is pressed along the vertical axis and flows in lateral direction into the cavity of the die. The commercial FE system Transvalor Forge 2007 was used to build up a model for the tool system and carry out the process simulation.

The schematic FE-model with the boundary conditions for the process of lateral extrusion is shown in Fig. 13. In order to keep the model complexity and the computational effort low, only a $45^{\circ}$-segment of the model was used. The punch and the ejector were modelled as rigid bodies. A hydrostatic pressure of $200 \mathrm{MPa}$ was applied to each surface element of the workpiece which is not in contact with the tools. By means of the FEA the form filling behaviour for the cold forging process with superimposed pressure was analysed [2].

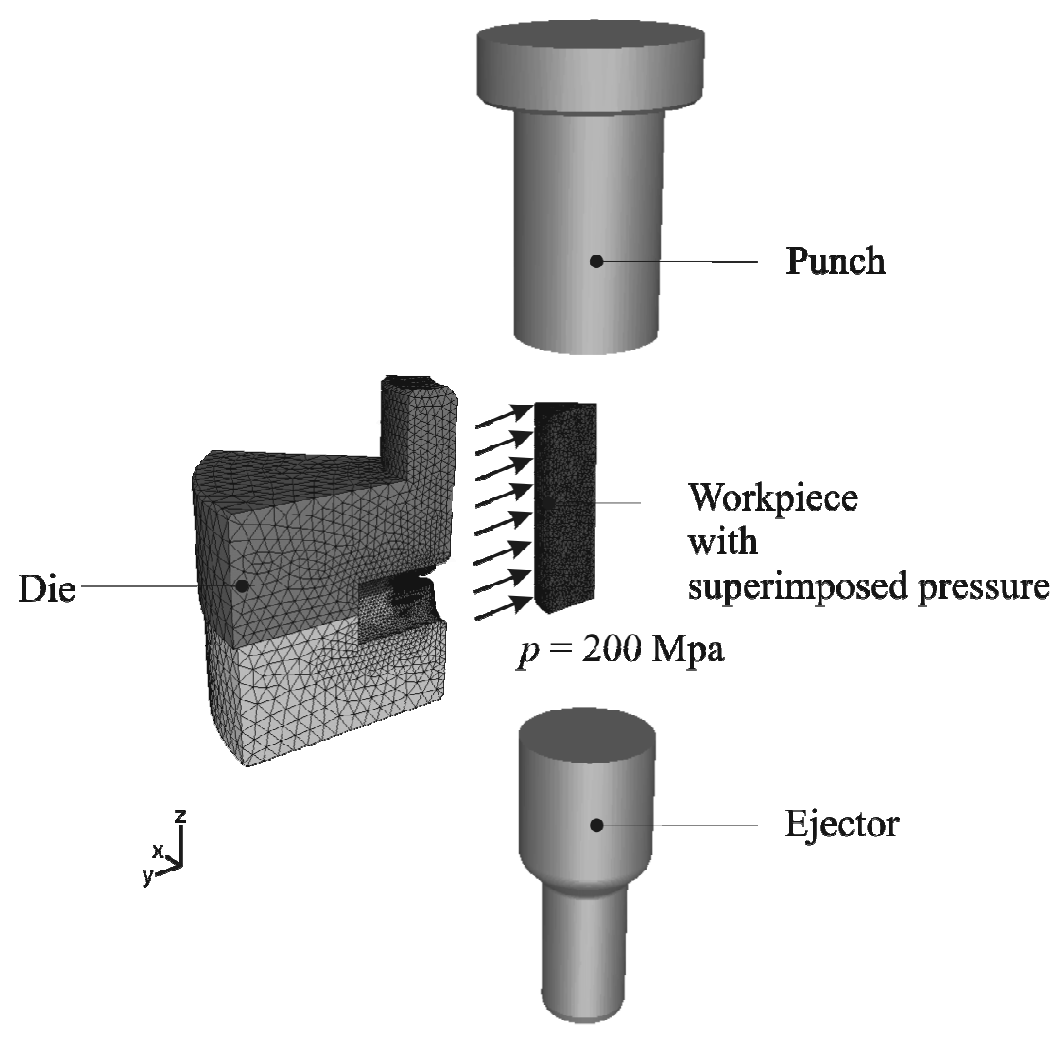

Fig. 13: FE-model with boundary conditions (cross geometry)

The tool concept shown in Fig. 1 offers the possibility to superimpose a maximum pressure of $200 \mathrm{MPa}$. By supplying an oil through various pipes, the superimposed pressure can be applied and maintained at the non-contacted surfaces during the forming operation. Due to the high hydrostatic pressure and the large amount of strain hardening high stresses arise in the tool components (Fig. 14). These peak stresses require the use of high-strength construction steels for the tools. 


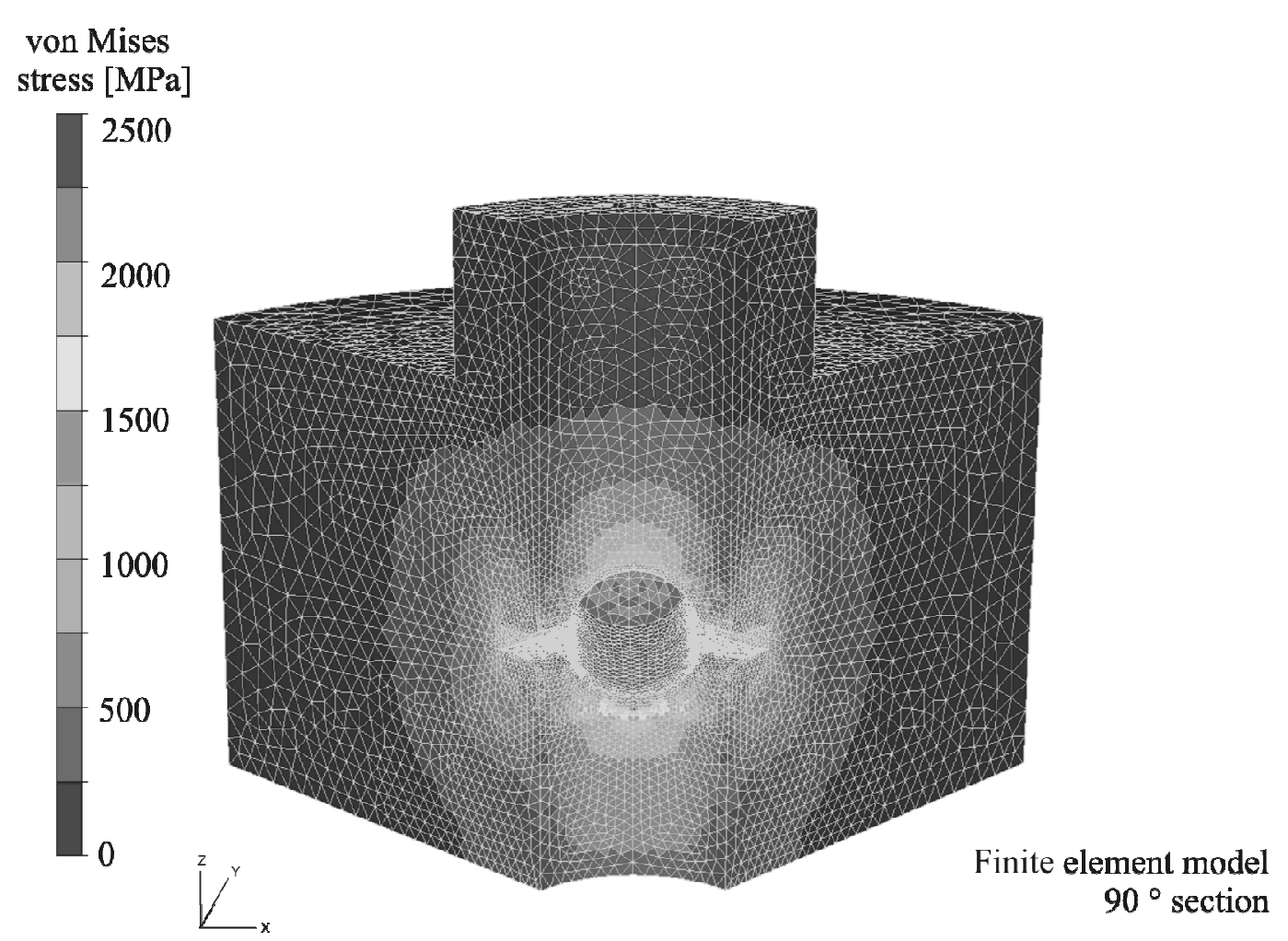

Fig. 14: Stresses in the die during forming of a cross-shaped part (AlZn5.5MgCu, EN AW-7075) with superimposed pressure

To simulate the loads of the complex tool shown in Fig. 3 an analysis of the closing plate has been done. The loads acting on the tool during the process are determined on the basis of FEA simulation results with the help of the FE package Abaqus/Standard 6.8-1. In order to save computation time and to simplify the complex simulation model only the closing plate has been considered in the FEA-based structural analysis, as this component is exposed to the highest service loads. In Fig. 15 a quarter model of the closing plate and the hydrostatic pressure acting on the inner tool surface is shown. The highest loads on the closing plate are applied in the ring section, as this tool area has the function to stop the punch movement.

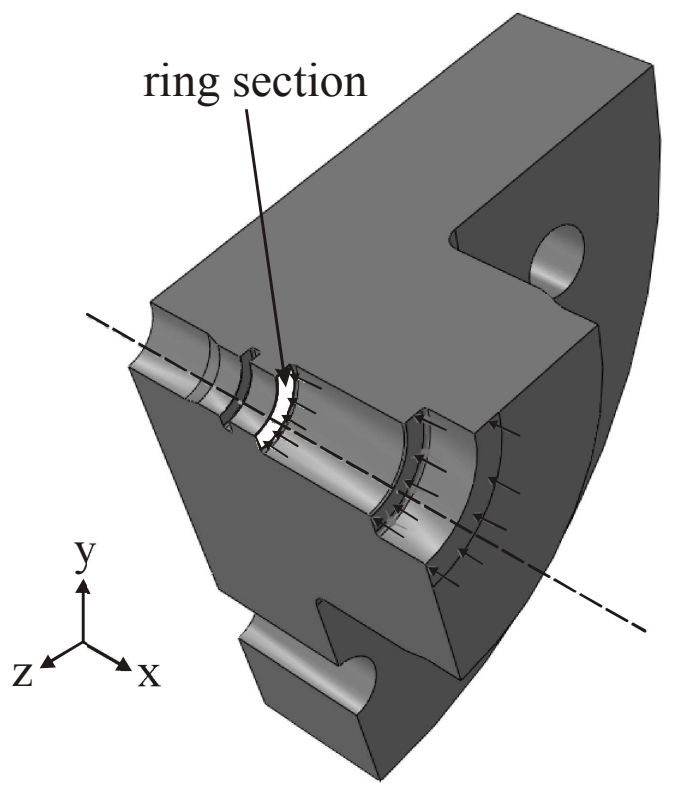

Fig. 15: Simulation model of the closing plate with hydrostatic pressure 
The boundary conditions for the analysis are defined as shown in Fig. 15. Tetrahedral elements with an average edge length of $6 \mathrm{~mm}$ were used for the spatial discretisation of the tool component. As can be seen in Fig. 16, the hydrostatic pressure leads to a maximum equivalent stress of $200 \mathrm{MPa}$ in section A and $800 \mathrm{MPa}$ in tool section $\mathrm{B}$.

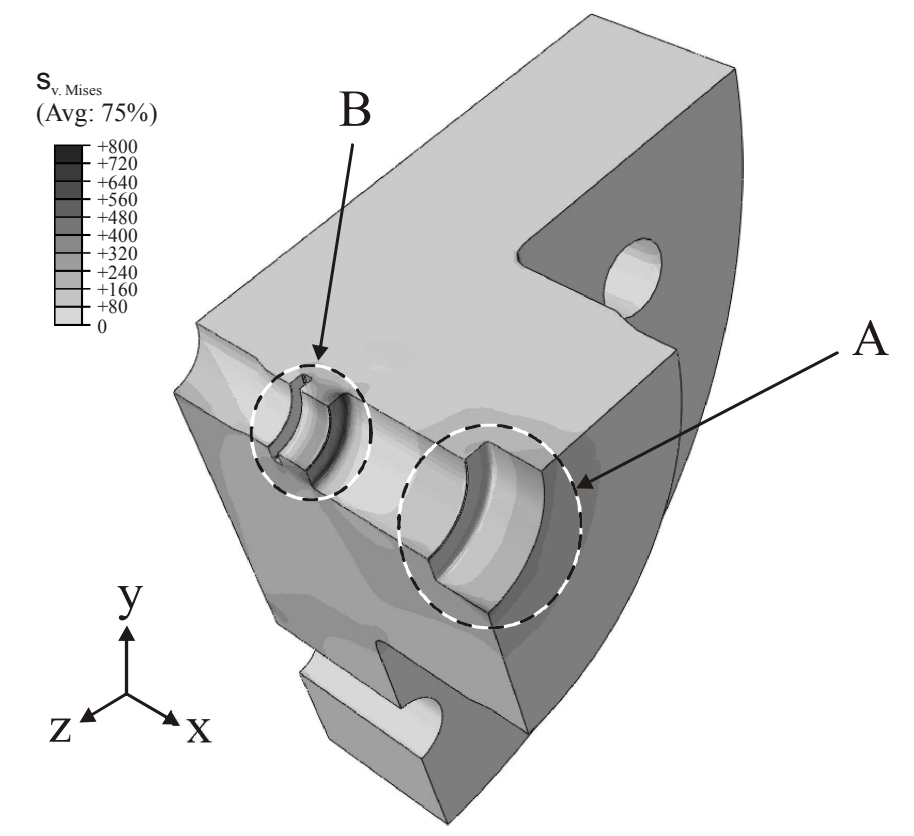

Fig. 16: FEA results of the closing plate under service load

\section{Prediction of damage by FEA}

Experimental investigations on the upsetting of the different aluminium alloys reveal that the workpiece is damaged with a growing upsetting range depending on the alloy. Therefore, different macro- and micromechanical models were tested and the results were compared to those from experimental investigations.

First a basic numerical model was built up in order to simulate the upsetting process for cylindrical billets of the alloys EN AW-6082 and EN AW-5083. The two-dimensional FE-model with the boundary conditions is shown in Fig. 17. The workpiece was modelled as an elasto-plastic component, whereas the dies were assumed to be rigid. For the numerical simulation the FEApackage Simufact.forming SFM has been used. The material data were not available in the database of the FE-software, so that material characterisations were also performed to obtain flow curves and further material properties for the simulation. The flow curves were implemented in the simulation models. In the following example, the flow curve data of the aluminium alloy AW-EN 5083 at ambient temperature were used to generate a mat-file for the use in the model. The effect of hydrostatic pressure is formulated in the FE-model by an edge load on each surface element of the workpiece. Here, the edge load is given by $0 \mathrm{MPa}, 50 \mathrm{MPa}$ and $85 \mathrm{MPa}$. The calculations with these pressures are equal to the pressure in the experimental observations presented before. 


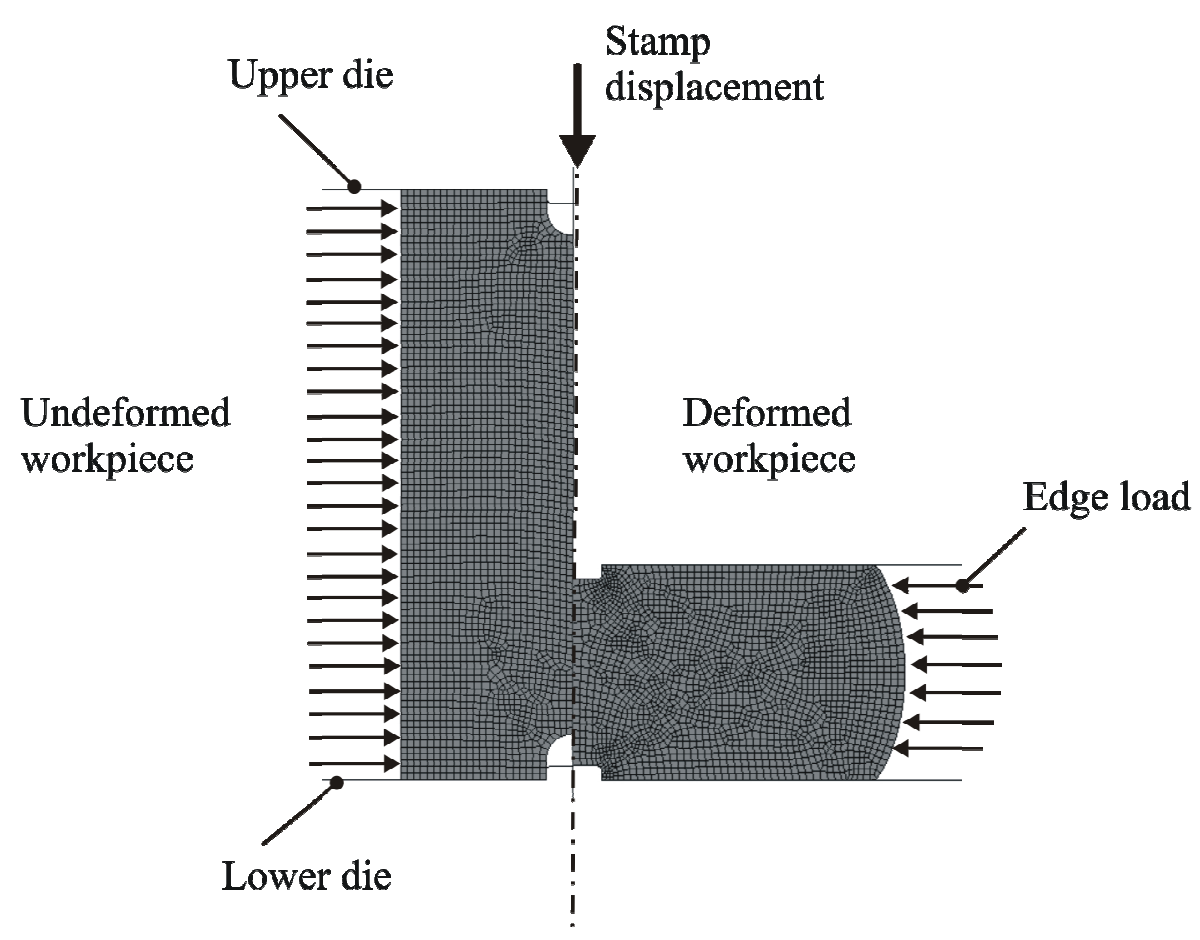

Fig. 17: FE-model (2-D) for the calculation of the damage prediction

Different mathematical approaches exist for the description and prediction of ductile damage as it may occur during forging operations. Current research concentrates on the use of macro- and micromechanical criteria for forming processes. On the one hand macromechanical models according to Brozzo [4], Cockcroft/Latham [6, 16], and Freudenthal [8] and on the other hand the micromechanical damage model of Rice/Tracey [18] and the model of Lemaitre, based on the effective stresses have been tested in this project. The macromechanical models as well as the micromechanical model represent the cumulative damage parameter using the integral of a stress function with respect to the equivalent plastic strain (Table 3).

Table 3: Macro- and micromechanical models for damage calculation

\begin{tabular}{|l|l|}
\hline Macromechanical models & \\
\hline$D_{\text {Brozzo }}=\int_{0}^{\varphi_{\mathrm{V}}} \frac{2 \sigma_{\mathrm{I}}}{3\left(\sigma_{\mathrm{I}}-\sigma_{\mathrm{m}}\right)} \mathrm{d} \varphi_{V}$ & \\
\hline$D_{\text {Cockcroft Latham }}=\int_{0}^{\varphi_{\mathrm{V}}} \max \left(\sigma_{\mathrm{I}}, 0\right) \mathrm{d} \varphi_{V}$ & \\
\hline$D_{\text {norm.Cockcroft Latham }}=\int_{0}^{\varphi_{\mathrm{V}}} \max \left(\frac{\sigma_{\mathrm{I}}}{\sigma_{\mathrm{V}}}, 0\right) \mathrm{d} \varphi_{V}$ & \\
\hline$D_{\text {Freudenthal }}=\int_{0}^{\varphi_{\mathrm{V}}} \sigma_{\mathrm{V}} \mathrm{d} \varphi_{V}$ & \\
\hline Micromechanical model & \\
\hline$D_{\text {Rice Tracey }}=\int_{0}^{\varphi_{\mathrm{V}}}\left(\frac{3}{2} \frac{\sigma_{\mathrm{m}}}{\sigma_{\mathrm{V}}}\right) \mathrm{d} \varphi_{V}$ & \\
\hline
\end{tabular}


For cold forging processes Landgrebe [13] proposed the use of the Model of Effective Stresses (MES) by Lemaitre [14]. It combines an evolution law for ductile damage with a reference value, making the criterion applicable to a wide range of problems involving damage. The material parameter identification is comparably simple. Just presented a different formulation of Lemaitre's damage model. In contrast to Landgrebe's formulation a crack closure factor is not used because a basic requirement for cumulative damage is crack growth which only occurs in case of positive triaxiality. The formulation of Lemaitre's model of effective stresses (MES) according to Just is available in the FE simulation software Simufact.forming.

\section{Modelling of damage evolution}

In coupled damage approaches, the mechanical properties of the workpiece are directly influenced by the damage evolution of the material. This reflects the fact that microscopic cracks cause a progressive decrease of the material strength. Most of these models consider the effective stress, which acts in the material with microscopic voids or cracks.

To predict damage in forging processes with active media, Lemaitre's MES was used in order to determine the forming limit. In this model the continuum is divided into Representative Volume Elements (RVE) as shown in Fig. 18. The RVE is loaded with external forces $F$, which cause the initiation of microscopic cracks due to the evolution and coalescence of voids in the material.

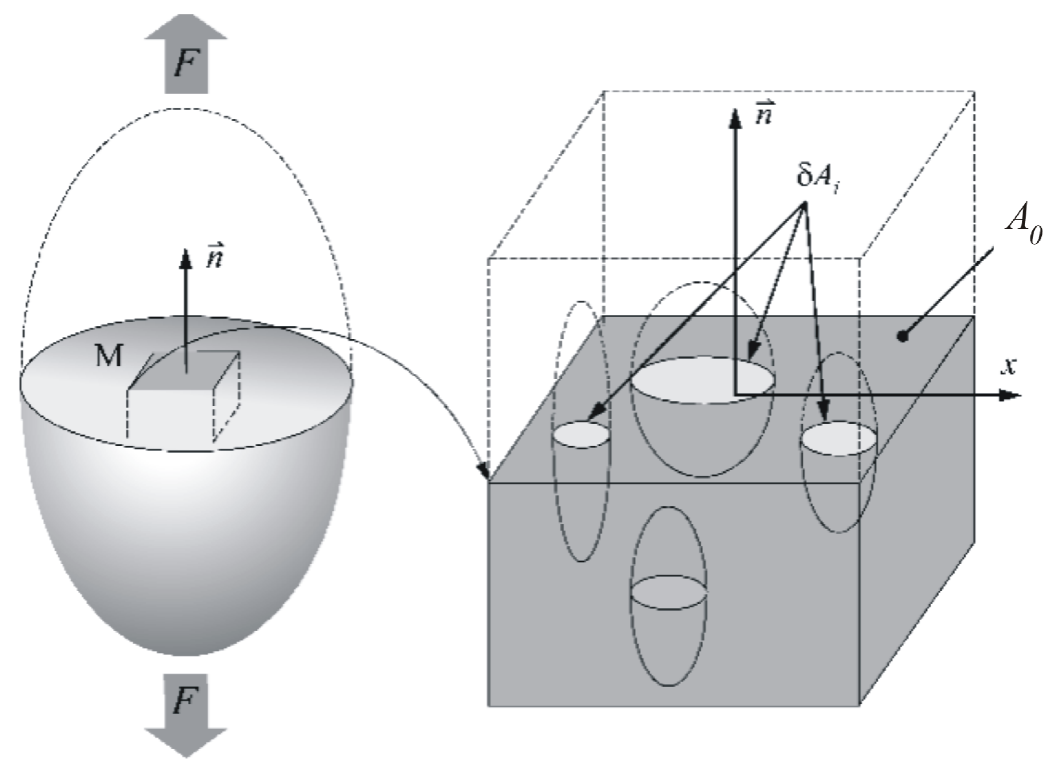

Fig. 18: Representative Volume Element (RVE) [12]

Thus, the effective load-carrying area decreases, and consequently, the effective stress $\tilde{\sigma}$ applied to this effective area increases as given in equation 6 . This model is based on the assumption that pores appear in the material under certain plastic deformation. If the material deforms further, the pores grow and coalesce, thus reducing the material cross-section that can carry the load.

$$
\widetilde{\sigma}=\frac{\vec{F}}{A}=\frac{\vec{F}}{A_{0}\left(1-\frac{\widetilde{A}}{A_{0}}\right)}=\frac{\sigma}{(1-D)}
$$

$D$ is the damage variable, which is 0 for the undamaged case and 1 for a fully damaged material. For the implementation in the FE-simulation a mathematical description of the evolution of the damage variable is needed. The evolution law for the absolute damage is (7): 


$$
d D=\frac{\sigma_{v}^{2} \cdot f(\eta)}{2 E S_{D}(1-D)^{2}} d \varepsilon_{e q}^{p}, 0 \leq D \leq 1
$$

Equation 7 depends on the von Mises equivalent stress $\sigma_{v}$. The value $\eta$ is the triaxiality, which takes into account triaxial stress states. The function $f(\eta)$ is implemented to calculate the damage in the current increment, where $\varepsilon_{e q}^{p}$ is the plastic strain. According to Lemaitre's model, the function $f(\eta)$ is proportional to the square of triaxiality as given in equation 8 , where $v$ is the Poisson's ratio.

$$
f(\eta)=\frac{2}{3}(1+v)+3(1-2 v)\left(\frac{\sigma_{m}}{\sigma_{v . \text { Mises }}}\right)^{2}
$$

The evolution of the damage variable $\partial D / \partial \varphi$ is developed with the help of tensile tests under uniaxial loading. In the beginning of the test the material is considered to be undamaged with $D=0$ and with the increasing deformation of the workpiece the value of the damage variable also increases. When it reaches the critical value $D_{1 c}$, the macroscopic damage in the workpiece initiates damage. The value of $D$ then increases until it reaches 1 and at this value of $D$ the material is considered as completely damaged. In order to calculate the critical value $D_{c}$ for triaxial stress states the following equation is used.

$$
D_{c}=\frac{D_{1 c}}{f(\eta)}\left(\frac{k_{f}^{\text {tensile }}\left(\varphi_{\text {crack }}^{p l}\right)}{\sigma_{v . \text { Mises }}}(1-D)\right)^{2} \leq 1
$$

Equation 9 represents a relation between the uniaxial tensile loading damage $D_{1 c}$ and the current triaxial stress states $\eta$, where $k_{f}^{\text {tensile }}\left(\varphi_{\text {crack }}^{\text {pl }}\right)$ is the fracture stress for uniaxial tensile tests which is also valid for compression tests since the flow curve remains the same, and the value of $D_{c}$ represents every increment in the simulation. By the relationship between the actual damage $D$ corresponding to $D_{c}$ a relative damage variable $D_{r e l}$ is found out as given in equation 10 .

$$
D_{r e l}=\frac{D}{D_{c}}
$$

This variable represents the damage state of the material under triaxial stress states.

\section{Material parameter determination for the MES}

The Method of Effective Stress (MES) by Lemaitre needs four material parameters for a reliable damage prediction. According to Just [12] a realistic prediction of the damage by using the MES is only valuable after a material characterisation has been accomplished on the same material batch.

In the following, the determination of material parameters for the MES is described. The essential damage values can be computed from values of the uniaxial stress-strain curve. The specifications of the tensile specimen are consistent with EN 10002, where the minimum diameter of the circular cross-section has to be $10 \mathrm{~mm}$.

$R_{\mathrm{m}}$ is the maximum stress in the tensile test at uniform elongation. Equivalent strain at uniform elongation $E_{\mathrm{p}, \mathrm{eq}, R \mathrm{~m}}$ is the corresponding strain at uniform elongation, used as a fracture initiation threshold. 
The critical damage value $D_{1 \mathrm{c}}$ at uniaxial load condition is taken by comparison of $R_{\mathrm{m}}$ and $R_{\mathrm{B}}$ from the stress-strain curve as well as the critical true strain $\varphi_{\mathrm{c}}$. Critical damage under uniaxial conditions denotes the general resistance of materials to ductile damage. According to Lemaitre's/Just's models, a linear slope is assumed after reaching uniform elongation. The damage resistance value $S_{\mathrm{D}}$ is computed from the assumed linear evolution of damage $D$ as derived in Eq. 11.

$$
S_{D}=\frac{R_{B}^{2}}{2 E\left(1-D_{1 c}\right)^{2} \frac{d D}{d \varepsilon_{e q}}}=\frac{R_{m}^{2}}{2 E \frac{d D}{d \varepsilon_{e q}}}
$$

In Table 4, the material damage parameters for the MES of the chosen aluminium alloys for the temperature of $20^{\circ} \mathrm{C}$ and strain rate $1 / \mathrm{s}$ are shown.

Table 4: Damage material parameters of aluminium alloys for the MES

\begin{tabular}{|c|c|c|c|c|c|}
\hline $\begin{array}{c}\text { Material number } \\
\text { EN AW: }\end{array}$ & $\begin{array}{c}\boldsymbol{R}_{\mathbf{p 0 , 2}} \\
{[\mathbf{M P a}]}\end{array}$ & $\begin{array}{c}\boldsymbol{R}_{\mathbf{m}, \mathbf{m}} \\
{[\mathbf{M P a}]}\end{array}$ & $\begin{array}{c}\boldsymbol{E}_{\mathbf{p}, \mathbf{e q}, \boldsymbol{R} \mathbf{m}} \\
{[\mathbf{\%}]}\end{array}$ & $\boldsymbol{D}_{\mathbf{1 C}}$ & $\boldsymbol{S}_{\mathbf{D}}$ \\
\hline 5083 & 182 & 333 & 24.8 & 0.1286 & 2.4982 \\
\hline 5754 & 99 & 237 & 31.1 & 0.2204 & 2.6423 \\
\hline $2017 \mathrm{~A}$ & 375 & 504 & 14.7 & 0.0382 & 1.2990 \\
\hline 6082 & 315 & 348 & 15.3 & 0.2459 & 3.3363 \\
\hline 7075 & 608 & 657 & 10.1 & 0.0221 & 0.8207 \\
\hline
\end{tabular}

For the calculation, the described relevant parameters were entered into the GUI of the used FEsoftware-system Simufact.forming SFM.

\section{Results of the numerical damage estimation}

The experimental damage evolution was shown before (Fig. 10 and Fig. 11) and is now compared to the numerical damage prediction with the different damage criteria. Here the results of the upsetting process (Fig. 17) with the aluminium alloy EN AW-6082 and the different damage criteria are shown. For the aluminium alloy EN AW-5083 the damage prediction by using Lemaitre's model is displayed for the pressures of $0 \mathrm{MPa}, 50 \mathrm{MPa}$ and $850 \mathrm{MPa}$ according to Fig. 11.

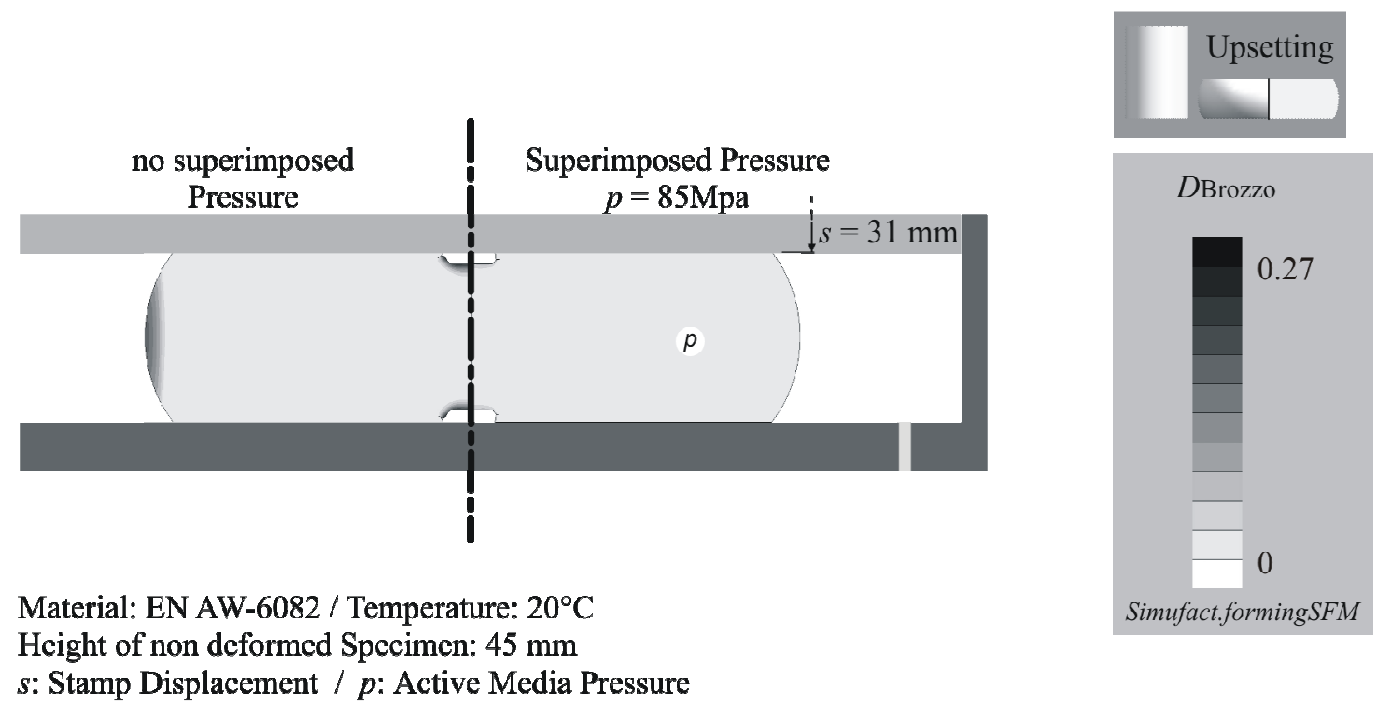

Fig. 19: Calculated damage for upsetting by using the model of Brozzo 


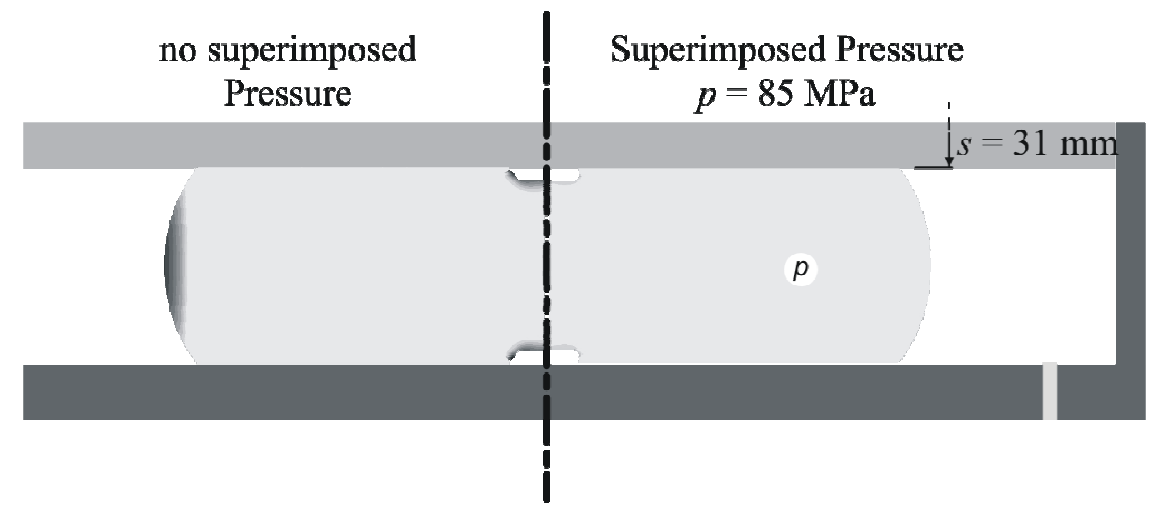

Material: EN AW-6082 / Temperature: $20^{\circ} \mathrm{C}$

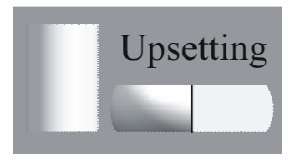

Height of non deformed Specimen: $45 \mathrm{~mm}$

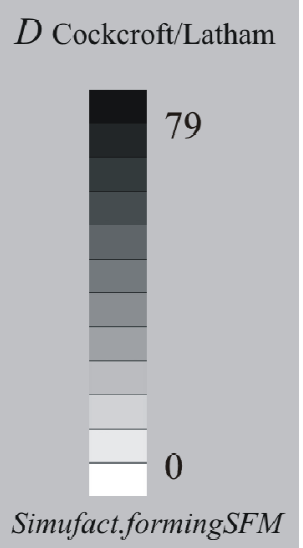

$s$ : Stamp Displacement / $p$ : Active Media Pressure

Fig. 20: Calculated damage for upsetting by using the model of Cockcroft/Latham

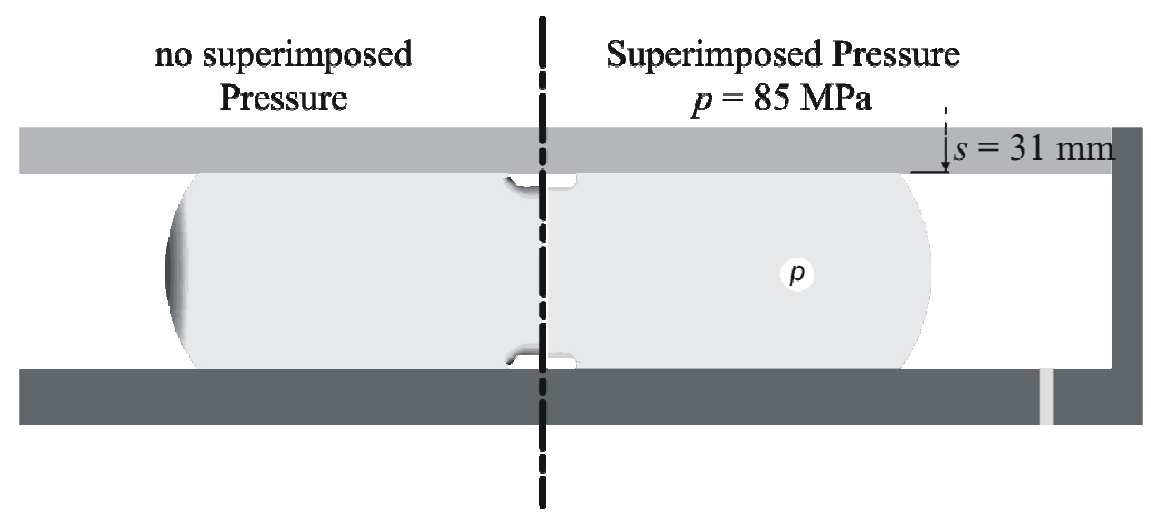

Material: EN AW-6082 / Temperature: $20^{\circ} \mathrm{C}$
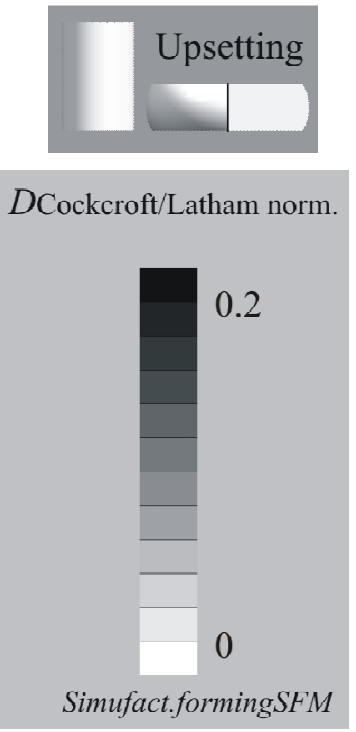

Height of non deformed Specimen: $45 \mathrm{~mm}$

$s$ : Stamp Displacement / $p$ : Active Media Pressure

Fig. 21: Calculated damage for upsetting by using the model of Cockcroft/Latham (norm.) 


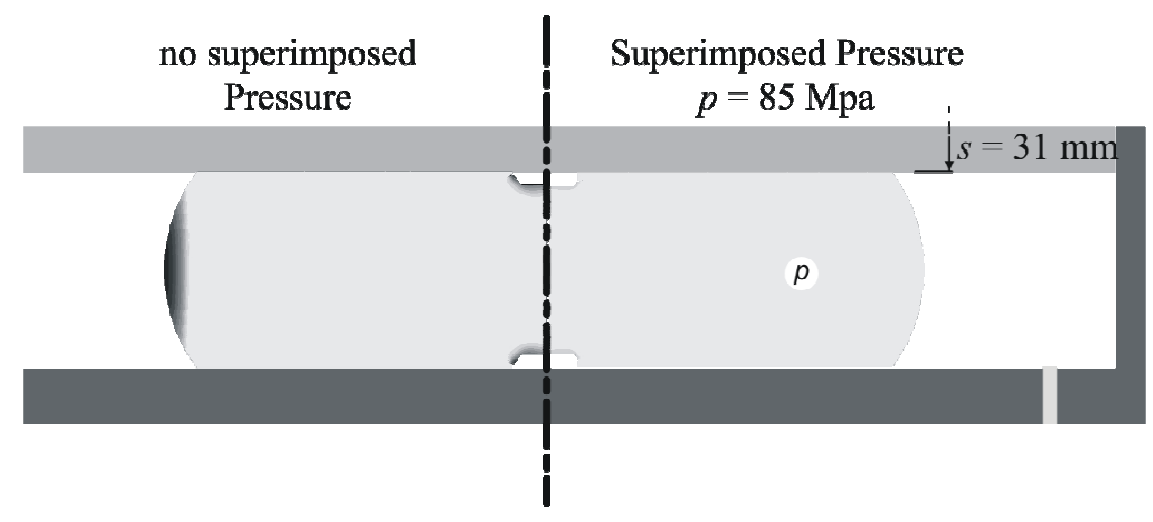

Material: EN AW-6082 / Temperature: $20^{\circ} \mathrm{C}$

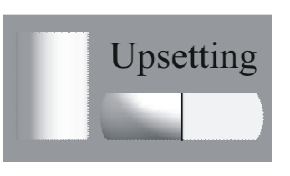

Height of non deformed Specimen: $45 \mathrm{~mm}$

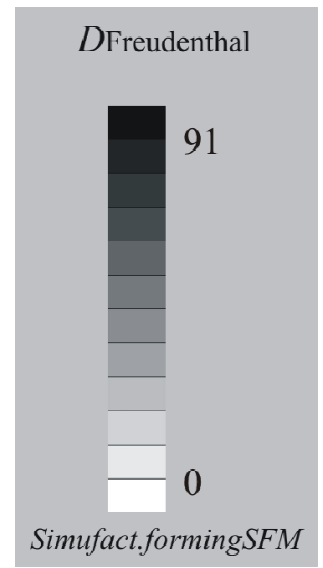

$s$ : Stamp Displacement / $p$ : Active Media Pressure

Fig. 22: Calculated damage for upsetting by using the model of Freudenthal

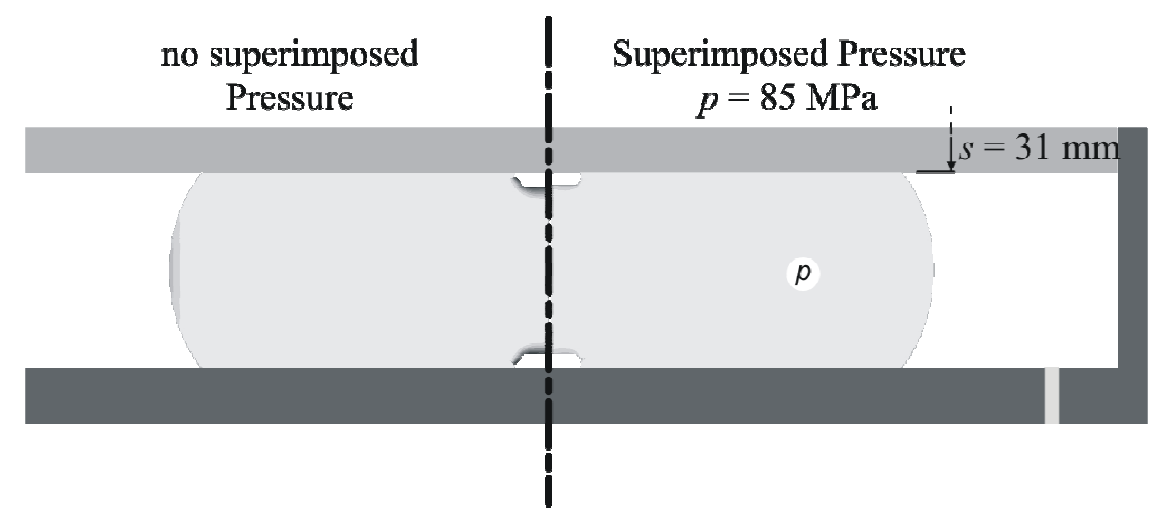

Material: EN AW-6082 / Temperature: $20^{\circ} \mathrm{C}$

Height of non deformed Specimen: $45 \mathrm{~mm}$

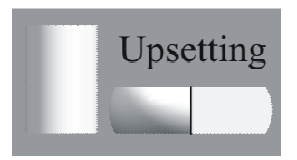

$s$ : Stamp Displacement / $p$ : Active Media Pressure

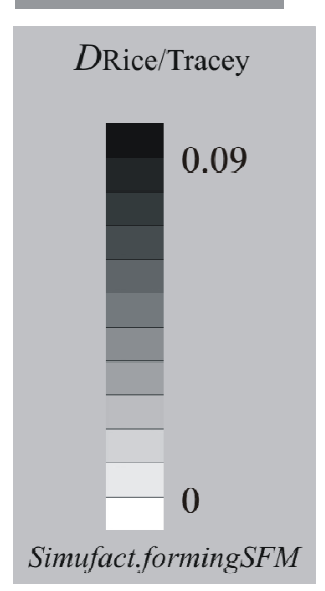

Fig. 23: Calculated damage for upsetting by using the model of Rice/Tracey

By the use of these models the damage evolution under superimposed pressure is shown as demonstrated before in the experimental observation. For the macromechanical models of Brozzo, Cockcroft and Latham etc. a critical value for the aluminium alloy can be given showing the damage of the workpiece. 


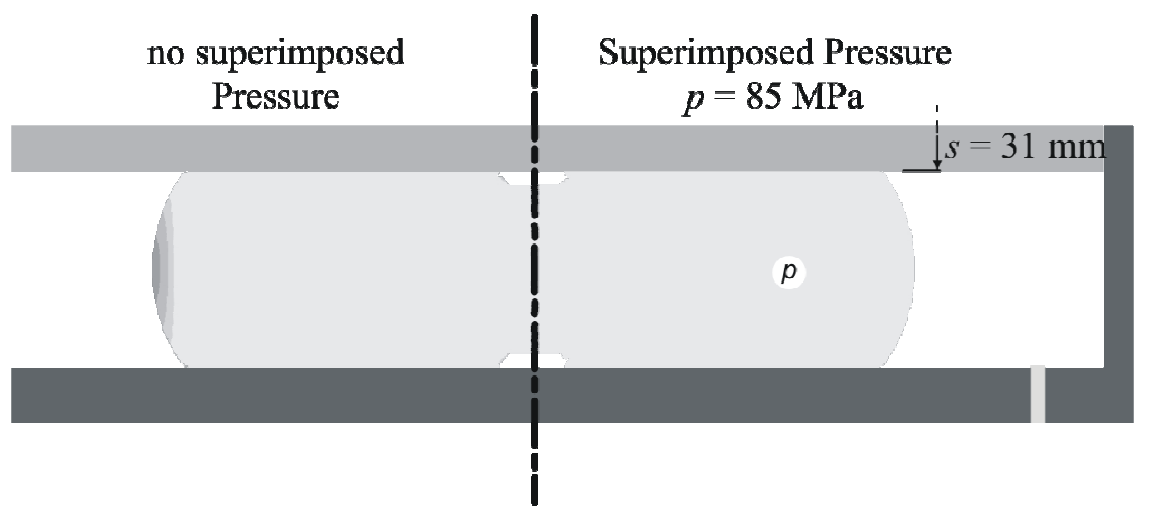

Material: EN AW-6082 / Temperature: $20^{\circ} \mathrm{C}$

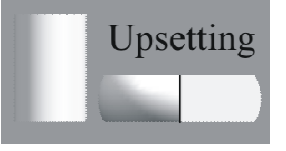

Height of non deformed Specimen: $45 \mathrm{~mm}$

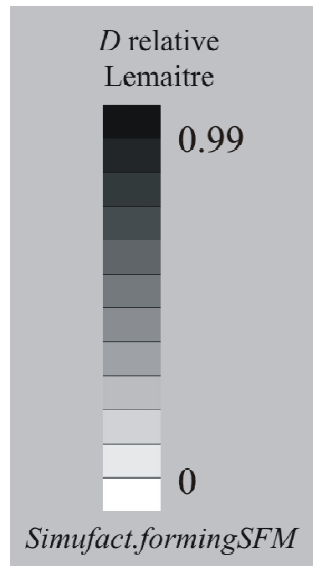

$s$ : Stamp Displacement / $p$ : Active Media Pressure

Fig. 24: Calculated damage for upsetting by using the model of Lemaitre

In Fig. 25 the forming limits of upsetting with and without superimposed pressure are displayed.

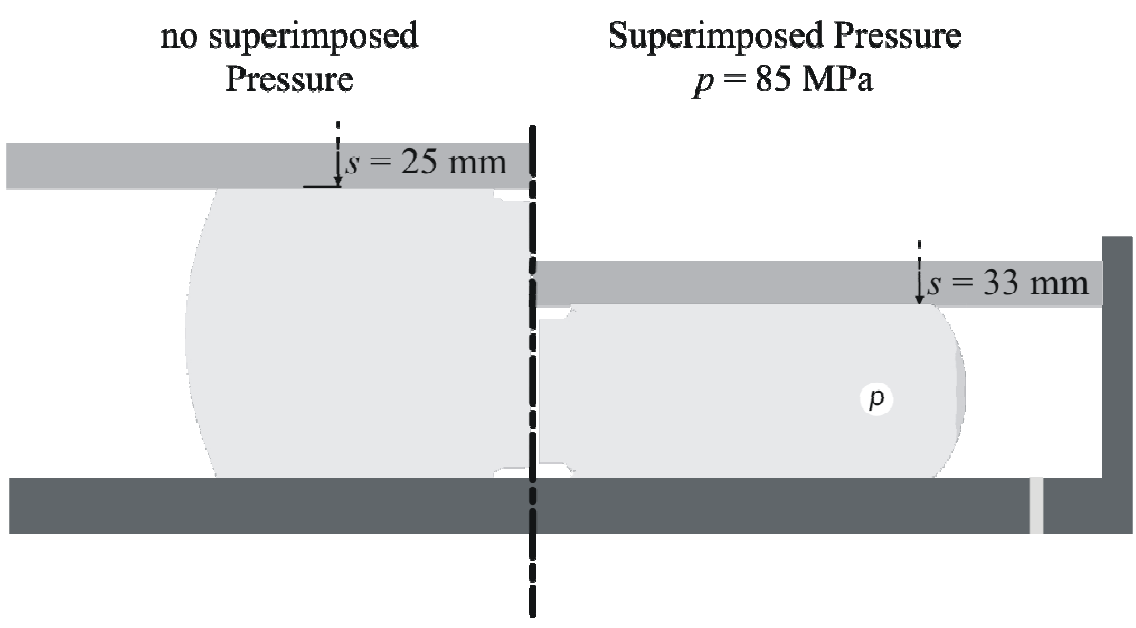

Material: EN AW-6082 / Temperature: $20^{\circ} \mathrm{C}$

Height of non deformed Specimen: $45 \mathrm{~mm}$

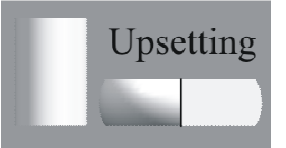

$s:$ Stamp Displacement / $p$ : Active Media Pressure

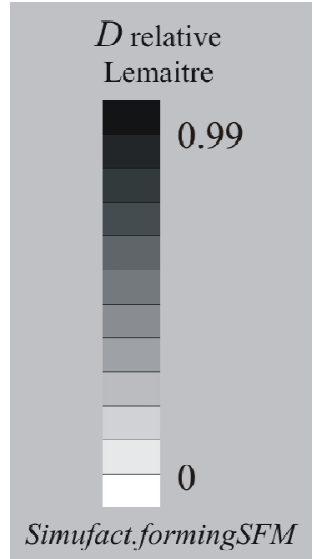

Fig. 25: Calculated damage for upsetting by using the model of Lemaitre

Lemaitre's damage model was used to describe the ductile damage in this process. Fig. 26 shows the damage values computed according to Lemaitre's model for the upsetting of the aluminium alloy EN AW-5083. The superimposed pressure was $0 \mathrm{MPa}, 50 \mathrm{MPa}$ and $85 \mathrm{MPa}$. The stamp displacement of $24 \mathrm{~mm}$ was chosen as an example to visualize the damage value.

It is obvious that without a superimposed pressure the stamp displacement of $24 \mathrm{~mm}$ shows damage in the workpiece. By a superimposed pressure of $85 \mathrm{MPa}$ the calculation according to Lemaitre's model shows no damage in the workpiece and the relative damage value increases (Fig. 26/bottom). 


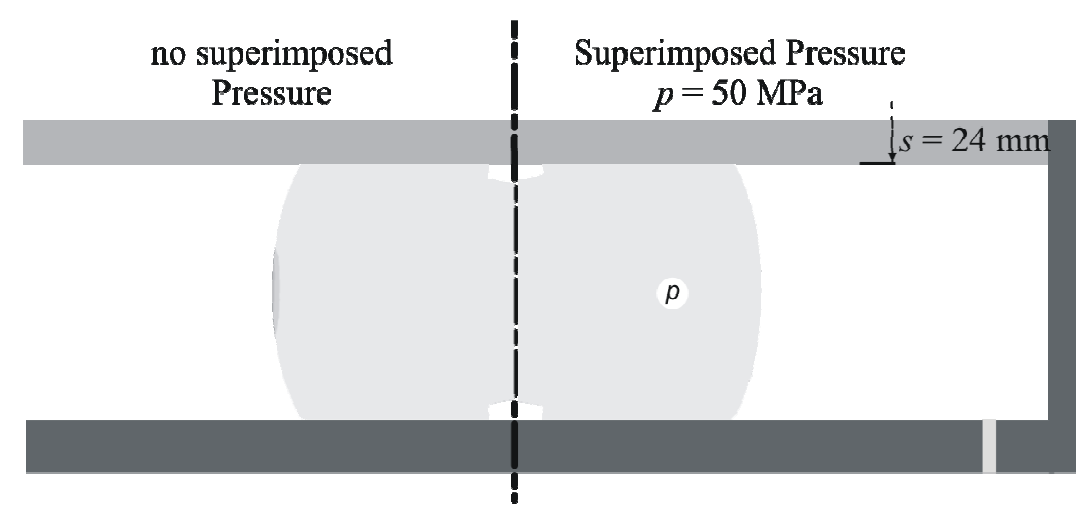

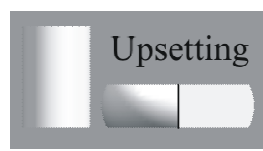

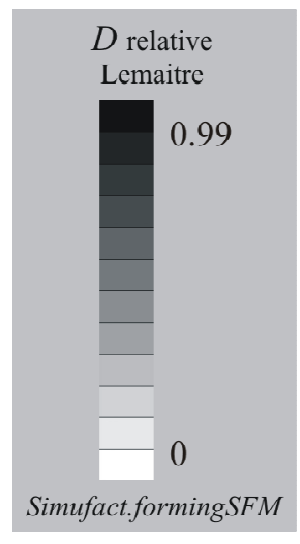

Material: EN AW-5083 / Temperature: $20^{\circ} \mathrm{C}$

Height of non deformed Specimen: $45 \mathrm{~mm}$

$s:$ Stamp Displacement / $p$ : Active Media Pressure

Fig. 26: Calculated damage for upsetting by using the model of Lemaitre (EN AW-5083)

Also the other demonstrated aluminium alloys were tested to show the forming limits by forming under superimposed hydrostatic pressure.

\section{Increase in Formability}

By means of the FEA the form filling behaviour for the cold forging processes under superimposed pressure and damage were analysed. In order to show the positive effect of increasing formability the hardness of the formed workpiece has been calculated. With the aid of the computed local strains and with the help of the yield stress determined from the material's flow curve, the hardness can be determined by the empirical correlation in Equation 12.

$$
H V \approx 1 / 3 \cdot k_{f}
$$

Comparing the Vickers hardness at different points in time for each geometry the following effect can be noticed. Contrasting the hardness distribution in the section of different geometries, the advantages of superimposed pressure forging are clearly visible.

By converting the demonstrated Vickers hardness $\mathrm{HV}$ by the correlation, that $\mathrm{HV} \approx 1.05 \mathrm{HB}$, the hardness after forging shown in Fig. 10 is according to Vickers hardness in Fig. 27. It can be shown that by geometry variation, here realised by upsetting to a defined limit, local hardening can be obtained. This can also be calculated by numerical simulation as shown in Fig. 27. 

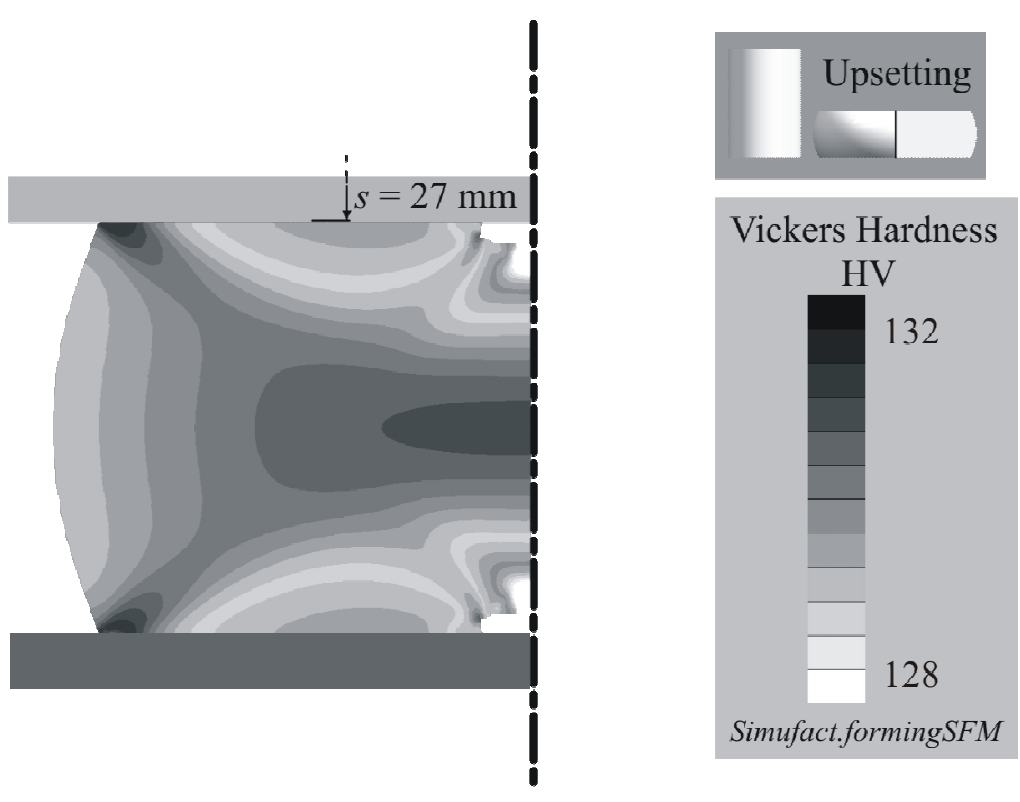

Material: EN AW-6082 / Temperature: $20^{\circ} \mathrm{C}$

Height of non deformed Specimen: $45 \mathrm{~mm}$ $s$ : Stamp Displacement

Fig. 27: Hardness distribution in the deformed cylinder (EN AW-6082)

Additional experimental results are necessary to validate the described approach.

Nevertheless, the positive effect on formability of superimposed hydrostatic pressure on cold forming processes can be displayed by FEA. Consequently, by applying damage models, FEA can be used in order to predict damage occurring within the cold forming process with superimposed hydrostatic pressure.

\section{Summary}

Forging processes offer a specific adaptation of local hardening according to the process parameters. Hence, it is possible to increase the strength and to realise strength gradients within the same forging part. With a subdivision of the component in reinforced and unreinforced areas, load adapted components can be realised according to the case of operation. At the Institute of Metal Forming and Metal-Forming Machines (IFUM) basic investigations in the field of local hardening of aluminium and metastable austenitic steel alloys by forging are carried out [9]. The superimposed pressure clearly contributed to enhancing the formability of aluminium alloys. By means of locally different degrees of deformation in the forging part a specific hardening is set. The work hardening can be increased compared to conventional forming processes. By means of the FEA the complex tool system was analysed to show the limits of stresses in the tools. Also the form filling behaviour and damage prediction for cold forging processes with superimposed pressure were analysed. Therefore, macro- and micromechanical damage models were used to calculate the damage prediction of an upsetting test with various aluminium alloys. For the model of Lemaitre the required material parameters for the different aluminium alloys have been adjusted before.

Furthermore, a local hardening of metastable austenitic steels by transformation-induced martensite was realised at IFUM. The results are also presented in this volume in the paper "Local strain hardening of metal components by means of martensite generation". Basing on these results the main focus of future research is on the development and the application of innovative tool concepts for forging under superimposed pressure at low temperatures as well as below ambient temperature. The objective is the production of aluminium as well as metastable austenitic steel 
components with adapted mechanical properties by local hardening for highly loadable structural components.

For the realisation of these objectives further expertise in the area of massive forming at low temperatures and in the field of tool cooling is nessecary. From the preliminary investigations it is known that low temperatures increase the transformation-induced martensite within forging parts made of metastable austenitic steels. Hence, the first step of future work is to upgrade the existing tool concept for massive forming with a cooling system. The advantage for the forging of aluminium alloys is a controlled temperature guidance of the tools and the pressure medium. Without cooling the medium temperature is supposed to increase by more than $100{ }^{\circ} \mathrm{C}$ during the application of the superimposed hydrostatic pressure at higher strain rates.

In addition to the continuation of the sucsessful aluminium forming with superimposed hydrostatic pressure the transfer of these results to metastable austenitic steels will be accomplished. The effect of local hardening by forming-induced martensite as well as the numerical analysis of the processes and their application are fields of interest.

As shown in the paper "Local strain hardening of metal components by means of martensite generation" within this volume, different local degrees of deformation during massive forming provide a specific influence on the phase transformation during the forming process. Besides, the degree of deformation, the strain rate, the forming temperature as well as the superimposed hydrostatic pressure are significant for the mechanical properties of the component after forging. By further research of the mechanisms influencing the martensite evolution during forging, the martensitic phase transformation can be controlled by local settings of the forming parameters.

The specific variation of the superimposed pressure during the forming process provides a control of the state of stress between compression and tensile. This results in a specific influencing of the martensite evolution in metastable austenitic steels. The damage-minimising effect of the superimposed pressure within aluminium components which has been examined so far is expanded to metastable austenitic steels and is complemented with the numerical investigation of a micromechanical damage.

Furthermore, a combined hot and cold forging process of metastable austenitic steels will be realised. The objective is the forming of complex geometries which are locally hardened by a martensitic phase transformation in a final cold forging step. To improve the component complexity a hot forming process is considered to produce preforms. In the final cold forging step the local solidifications are inserted in the component.

In addition, investigations are carried out for the surface structuring of components made of metastable austenitic steels. Therefore, different structural geometries and their influence on the local hardening are examined. Besides, the use of cooled tools will increase the phase transformation in spite of low degrees of deformation.

For the numerical analysis of the phase transformation, the implementation of the developed routines in the commercial FE-software system FORGE will be done.

From the state shown above and the preliminary investigations the following partial objectives are derived:

- Expansion of the process limitations within work hardening of aluminium and steel in particular in the area of complex component structures

- Development of tool concepts for forging with and without superimposed hydrostatic pressure at low temperatures as well as below ambient temperature

- Obtaining higher degrees of forming by warm and subsequent cold forging

- Increasing component strength by surface structures in highly loaded areas

- Basic understanding of the influence of the superimposed hydrostatic pressure on martensitic structures 


\section{Acknowledgement}

The authors would like to thank the German Research Foundation (DFG) for funding this Collaborative Research Center 675 (SFB 675) - "Creation of high strength metallic structures and joints by setting up scaled local material properties". The dedicated subproject B2 is called "Selective strain hardening of structure components by pressure imposed cold bulk forming".

\section{References}

[1] A. Behrens, H. Just: Entwicklung von Strategien zur Erweiterung der Formgebungsgrenzen komplexer Kalt- und Halbwarmmassivumformvorgänge auf Grundlage der Schädigungsmechanik mit FE-Methoden, Zwischenbericht zum Forschungsvorhaben Be 965/75 im Rahmen des Schwerpunktprogrammes der Deutschen Forschungsgemeinschaft: Erweiterung der Formgebungsgrenzen bei Umformprozessen, Univ. d. Bundeswehr, Inst. f. Konstruktions- u. Fertigungstechnik, Hamburg, Germany, (2003)

[2] B.-A. Behrens, T Hagen, F. Meiners, S. Röhr: Gesenkschmieden von hochfesten Aluminiumlegierungen mit überlagerten Druckspannungen, Abschlussbericht zum DFG-Schwerpunktprogramm 1074, pp. 159-166, DFG Deutsche Forschungsgemeinschaft, Hrsg. R. Kopp, Aachen, Germany, (2005)

[3] B.-A. Behrens, I. Pfeiffer: Massivumformung von Magnesiumlegierungen. Entwicklung eines Werkzeugsystems zum Schmieden mit überlagertem hydrostatischen Druck, wt Werkstattstechnik online, Band 99, Heft 10; Germany, (2009)

[4] P. Brozzo, B. De Luca, R. Renclina: A new method for prediction of the formability limits of metal sheets; Proceeding of the $7^{\text {th }}$ Biennial Congress of International Deep Drawing Research Group, (1972)

[5] A. H. Clausen, et al.: Flow and fracture characteristics of aluminium alloy AA5083-H116 as function ofstrain rate, temperature and triaxiality. In: Materials Science and Engineering A, Num. 364, V. 1-2, (2004), pp. 260-272.

[6] M.G. Cockcroft, D.J. Latham: Ductility and the workability of metals; Journal of the Institute of Metals 96, (1968)

[7] I. El Galy, I.Pfeiffer,B.-A. Behrens: Application of Acoustic Emission to Monitor Forging Processes of Magnesium Alloys under Superimposed Hydrostatic Pressure; Material Science and Technology 2009 (MS\&T09); Pittsburgh, USA; 23-29 Oct. (2009)

[8] A. Freudenthal: The inelastic behaviour of engineering metals and structures, Wiley, New York, (1950)

[9] S. Hübner, et al.: Im Fertigungsprozess lassen sich Bauteile spezifisch optimieren, MM Maschinenmarkt, Ausgabe 44, Germany, 26. Oktober 2009, pp. 26 - 2

[10] S. P.Joshi, et al.: On the Occurrence of Portevin-Le Châtelier Instabilities in Ultrafine-Grained 5083 Aluminum Alloys, in: Experimental Mechanics, Volume 49, Number 2, Springer Boston, USA, April 2009, pp. 207-218

[11]H. Just, A. Behrens: Investigations on the use of damage indicating criteria in FE simulations of cold and semi-hot bulk forging operations, Proceedings of IMECE, Anaheim, CA, (2004)

[12]H. Just: Erweiterung der Formgebungsgrenzen rissbehafteter Halbwarm-Massivumformprozesse unter Anwendung der Schädigungsmechanik und der Finite Elemente Methode, Berichte aus dem Institut für Konstruktions- und Fertigungstechnik, Dissertation, Hamburg, Aachen, Germany, (2005) 
[13]D. Landgrebe: Bestimmung der Formänderungsgrenzen durch duktiles Werkstoff-versagen bei Kaltumformverfahren mit Hilfe der FEM, Dr.-Ing. thesis, (2000)

[14] J. Lemaitre: A Course on Demage Mechanics, $2^{\text {nd }}$ Ed., Springer Verlag, Berlin, (1996)

[15]F. Meiners: Untersuchungen zur wirkmedienbasierten Massivumformung von Aluminiumwerkstoffen, Dissertation, Universität Hannover, Germany, (2004)

[16] S.I. Oh, S. Kobayashi, C.C. Chen: Ductile fracture in axisymmetric extrusion and drawing, Journal of Engineering for Industry 101, (1979)

[17] O. Pawelski: „Hydrostatisches Stauchen - ein neues Verfahren zur Formgebung schwer umformbarer Werkstoffe“, Technik und Forschung, 170, pp. 682-686, Germany, (1967)

[18] J.R. Rice, D.M. Tracey: On the ductile enlargement of voids in triaxial stress fields; Journal of Mechanics and Physics of Solids 17, (1969) 